\section{SANDIA REPORT}

SAND88-0108 $-\mathrm{UC}-34$

Unlimited Release

Printed April 1988

\title{
A Comparison of Analytical Techniques for Modeling the Rayleigh-Taylor Response of Elastic-Plastic Solids
}

\author{
Allen C. Robinson
}

Prepared by

Sandia National Laboratories

Albuquerque, New Mexico 87185 and Livermore, California 94550

for the United States Department of Energy

under Contract DE-AC04-76DP00789

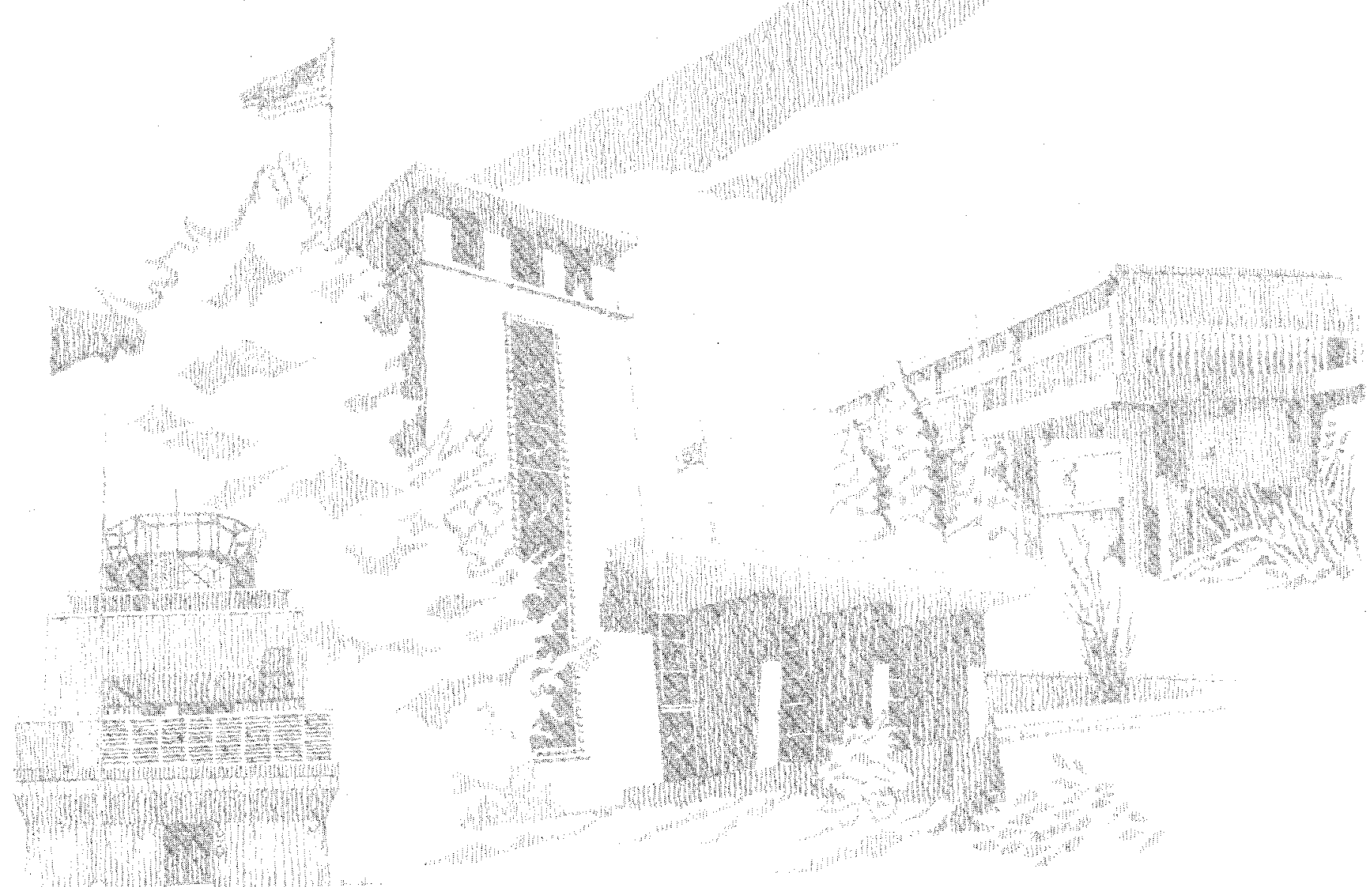


Issued by Sandia National Laboratories, operated for the United States Department of Energy by Sandia Corporation.

NOTICE: This report was prepared as an account of work sponsored by an agency of the United States Government. Neither the United States Government nor any agency thereof, nor any of their employees, nor any of their contractors, subcontractors, or their employees, makes any warranty, express or implied, or assumes any legal liability or responsibility for the accuracy, completeness, or usefulness of any information, apparatus, product or process disclosed, or represents that its use would not infringe privately owned rights. Reference herein to any specific commercial product, process, or service by trade name, trademark, manufacturer, or otherwise, does not necessarily constitute or imply its endorsement, recommendation, or favoring by the United States Government, any agency thereof or any of their contractors or subcontractors. The views and opinions expressed herein do not necessarily state or reflect those of the United States Government, any agency thereof or any of their contractors.

Printed in the United States of America

Available from

National Technical Information Service

U.S. Department of Commerce

5285 Port Royal Road

Springfield, VA22161

NTIS price codes

Printed copy: A03

Microfiche copy: A01 
Distribution

Category UC-34

SAND88-0108

Unlimited Distribution

Printed April 1988

\title{
A Comparison of Analytical Techniques for Modeling the Rayleigh-Taylor Response of Elastic-Plastic Solids
}

\author{
Allen C. Robinson \\ Computational Physics \& Mechanics Division II
}

\begin{abstract}
The Rayleigh-Taylor instability response of elastic-plastic solids can be modeled by approximate means. However, neither the linear elastic response equation nor the so-called "minimum amplituce" instability criterion derived from rigid plastic analyses is sufficient to describe overall stability characteristics at very large driving pressures. We compare several versions of the modal or one-degree-of-freedom approximation technique and indicate the common features which must be included to obtain the qualitative response seen in numerical experiments.
\end{abstract}




\section{Contents}

$\begin{array}{ll}\text { 1. Introduction } & 1\end{array}$

2. The Modal Technique and the Constitutive Equations 5

2.1 Application to a Newtonian Fluid and an Elastic Solid Layer . . . . 7

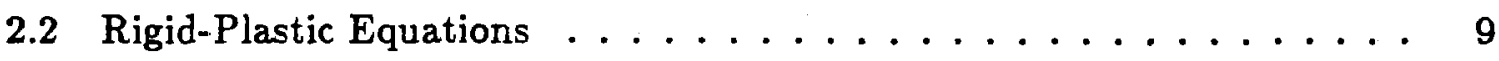

3. Elastic-Plastic Modal Analyses $\quad 10$

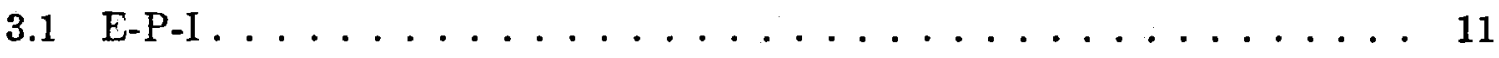

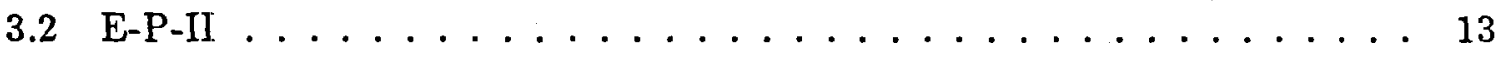

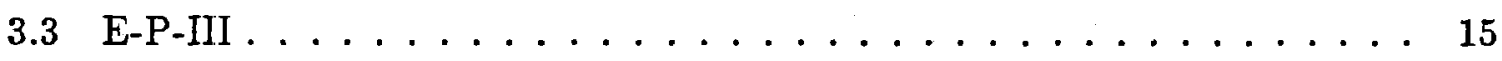

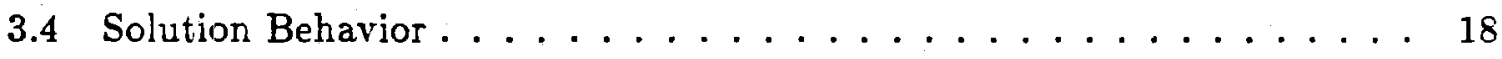

$\begin{array}{lr}\text { 4. Discussion } & 20\end{array}$

5. References $\quad 21$ 


\section{List of Figures}

1 Rayleigh-Taylor instability of an elastic-plastic plate. . . . . . . . . 22

2 Phase plane for the rigid-plastic one-degree-of-freedom equation. . . . . 23

3 Growth rate comparison between the modal approximation and the exact solution for a Newtonian fluid. . . . . . . . . . . . . . 24

4 Growth rate comparison between the modal approximation and the exact solution for an elastic fluid. . . . . . . . . . . . . . 25

5 Limit stress comparison between the E-P-II model and the rigid plastic model. ...................... 26

6 Amplitude $q$, velocity $\dot{q}$ and stress variable $s$ for $\mathrm{E}-\mathrm{P}$-II with $\lambda / h=4$ and $p_{m}=60 \mathrm{GPa}$ at various initial amplitudes. . . . . . . . . 27

7 Amplitude $q$, velocity $\dot{q}$ and stress variable $s$ for E-P-II and various

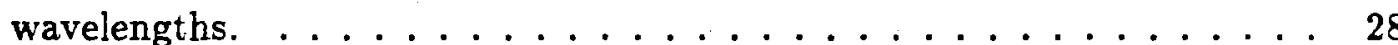

8 E-P-II unstable solutions for $p_{m}=10 \mathrm{GPa}(\square), 60 \mathrm{GPa}(x)$ and 120 $\mathrm{GPa}(+)$. Each point represents three distinct calculations. . . . . . . . 29

9 Comparison between E-P-II model predictions and data of Barnes, et al. 30

10 Amplitude $q$, velocity $\dot{q}$ and equivalent stress variables $s$ for the different elastic-plastic models in an oscillatory response regime. . . . . . . . 31

11 Amplitude $q$, velocity $\dot{q}$ and equivalent stress variables $s$ for the different elastic-plastic models in an unstable response regime. . . . . . . . 32 


\section{Nomenclature}

\begin{tabular}{|c|c|c|}
\hline$c$ & - & $e^{-k y}$ \\
\hline$g$ & - & Equivalent body force acceleration \\
\hline$h$ & - & Nominal plate thickness \\
\hline$k$ & - & Spanwise perturbation wavenumber \\
\hline$n$ & - & Perturbaton growth rate \\
\hline$p$ & - & Pressure \\
\hline$p_{0}$ & - & Driving pressure on plate \\
\hline$p_{m}$ & - & Maximum value of $p_{0}$ \\
\hline$q$ & - & Maximum perturbation amplitude of interface $\eta_{1}$ \\
\hline$s$ & - & Deviatoric stress variable (scalar) \\
\hline$s_{1}$ & - & von-Mises yield stress in pure shear \\
\hline$s^{*}$ & - & Limiting value of $s$ \\
\hline$\tilde{\mathbf{s}}(\mathrm{n})$ & - & Boundary traction vector \\
\hline$t$ & - & time \\
\hline$t_{x z}, t_{y y}, t_{x z}, t_{x y}$ & - & Non-zero components of Cauchy stress tensor, $\mathbf{T}$ \\
\hline $\mathbf{v}$ & - & Assumed velocity field $=\left(v_{x}, v_{\nu}\right)=\left(\dot{q} e^{-k \nu} \sin k x, \dot{q} e^{-k \nu} \cos k x\right)$ \\
\hline $\bar{x}$ & - & $\mathbf{S} \cdot \mathbf{M} /\left(2 s_{1}\right)$ \\
\hline$G$ & - & Elastic shear modulus \\
\hline$Y$ & - & von-Mises yield stress in uniaxial tension \\
\hline$Y_{h}$ & - & Value of $Y$ on free surface $h$ \\
\hline$\dot{W}$ & - & Stress power $=\mathbf{S} \cdot \mathbf{D}$ \\
\hline$\alpha$ & - & $d Y / d p$ \\
\hline$\beta$ & - & Coefficient of $\mathbf{S}$ in Prandtl-Reuss relation \\
\hline$\gamma$ & - & $1-e^{-2 k h}$ \\
\hline$\eta, \eta_{0}, \tilde{\eta}$ & - & $2 G k q / s_{1}, 2 G k q(0) / s_{1}, \eta-\eta_{0}$ (respectively) \\
\hline$\eta_{1}, \eta_{2}$ & - & Driven face and free face interfaces, respectively. \\
\hline$\lambda$ & - & Spanwise perturbation wavelength \\
\hline$\mu$ & - & Newtonian viscosity \\
\hline$\nu$ & - & Newtonian kinematic viscosity \\
\hline$\rho$ & - & Density \\
\hline $\mathbf{D}$ & - & Rate of strain tensor $=\left(\nabla \mathbf{v}+\nabla \mathbf{v}^{T}\right) / 2$ \\
\hline $\mathbf{M}$ & - & $\mathrm{D} / \dot{q} k$ for $\mathrm{v}$ defined above \\
\hline $\mathbf{M} \cdot \mathbf{M}$ & - & $2 c^{2}$ \\
\hline $\mathbf{S}$ & - & Deviatoric stress tensor with components $s_{i j}$ \\
\hline $\mathbf{T}$ & - & Cauchy stress tensor \\
\hline
\end{tabular}




\section{Introduction}

When a high density material is accelerated by a low density material in the direction of the positive density gradient, a classical Rayleigh-Taylor instability may develop along the interface between the two materials. The problem has been studied for some time in the case of fluids and the literature is voluminous (Sharp, 1984). We wish to study aspects of this instability behavior when the high density material is an elasticplastic solid pushed by a low density gas. We are interested principally in the regime where the driving pressure of the gas is much greater than the yield strength of the solid and wish to investigate how the properties of elasticity and plasticity for the solid plate may assist in delaying or suppressing the onset and growth of unstable modes.

Miles (1966) provided analytical estimates of the effect of solid properties on the growth of Rayleigh-Taylor instabilities. Using an energy integral argument and employing the inviscid fluid Rayleigh-Taylor eigenfunctions as basis functions for the velocity field, he determined two types of stability equations for two different regimes in the solid response of an incompressible elastic-plastic plate. The plate was assumed to be of thickness, $h$, with elastic shear modulus, $G$, and von-Mises yield stress in pure shear, $s_{1}$, which is related to the yield stress in unaxial tension, $Y$, by $Y=s_{1} \sqrt{3}$ (see Figure 1). A simple pressure boundary condition, $p_{0}$, was applied on the lower surface of the plate. He examined two limiting cases. The first case was for infinitesimal perturbations about an assumed anisotropic state of stress in the plate given by the deviatoric stress components $s_{i j}=\left(\delta_{i j}-3 \delta_{i 2} \delta_{j 2}\right) s_{1} / \sqrt{3}$. Thus $s_{i j} s_{i j}=2 s_{1}^{2}$ so that the stress state was assumed to lie on the yield surface. For perturbation amplitudes much smaller than $h s_{1} / p_{0}$, Miles obtained, using the Prandtl-Reuss constitutive law, a perturbation amplitude equation of the form

$$
\ddot{q}-\left(g k-\frac{13}{4} \frac{G}{\rho} k^{2}\right) q=0
$$

where $q$ measures the amplitude of the perturbation, the dot indicates a time derivative, $g$ is the equivalent body force acting on the plate, $k$ is the wavenumber of the spanwise perturbation and $\rho$ is the density. Since $p_{0}=\rho g h$, this implies that there exists a wavelength of maximum growth rate, $\lambda_{m}$, given by $\lambda_{m} / h=13 \pi G / p_{0}$. Thus, if the driving pressures are on the order of the shear modulus, the wavelength of maximum growth rate is roughly an order of magnitude larger than the thickness of the plate. A cutoff wavelength which is exactly half of the most unstable wavelength is also predicted. All wavelengths smaller than this cutoff wavelength would be predicted to be stable. For many metals this would imply that, unless the pressures driving the plate are on the order of $100 \mathrm{GPa}$ or more, the Rayleigh-Taylor response can be neglected 
as far as plate integrity is concerned provided initial perturbations are small. In order to provide an estimate for larger amplitudes, Miles assumed the Levy-Mises rule for plastic flow and using the same modal technique determined an equation of the form

$$
\bar{q}-g k q=-\frac{4 k s_{1}}{\rho\left(1+e^{-k h}\right)} \operatorname{sgn} \dot{q}
$$

where sgn $\dot{q}$ indicates the sign of $\dot{q}$. If one scales time on the instability growth rate for a perfect fluid, $\tau=\sqrt{g k} t$, and the perturbation amplitude by $h$, then if we define $f(\tau)$ by $q / h=\delta f(\tau)$, where $\delta=4\left(1+e^{-k h}\right)^{-1} s_{1} / p_{0}$, one obtains

$$
f^{\prime \prime}(\tau)-f(\tau)=-\operatorname{sgn} f^{\prime}(\tau)
$$

or, in integrated form,

$$
f^{\prime 2}-\left(f-\operatorname{sgn} f^{\prime}\right)^{2}=f_{0}^{\prime 2}-\left(f_{0}-\operatorname{sgn} f_{0}^{\prime}\right)^{2} .
$$

The level curves of Equation 4 (i.e., the trajectories of Equation 3) are shown in Figure 2. For regions near the origin in the phase plane the motion is stable in the sense that the flow lines do not go to infinity for any values of the initial state. However, for large enough values of either $f(\tau)$ or $f^{\prime}(\tau)$ the motion may follow an unstable trajectory. In particular, if one assumes $\dot{q}=0$ at the beginning of the motion one concludes that any initial $|q|>\delta h(|f|>1)$ will lead to an unstable motion while any $q$ less than this cutoff value will be stable. Thus the parameter, $\delta$, measures the minimum amplitude of an initial perturbation (in plate thickness units) which must be present for instability and has a magnitude of the order of the yield strength divided by the driving pressure. The scaling employed above and the small amplitude assumptions inherent in the modal analysis technique imply that we are examining the regime where $q / h \sim \delta \ll 1$. Miles' analysis is the first known discussion of a minimum amplitude criterion for instability given an assumption of rigid-plastic material behavior.

White (1973), in an informal report, discussed a unified analytical technique which included both the elastic and plastic response behavior seen in Miles' equations. White assumed that the whole flow was on the yield surface throughout the motion. Thus no explicit time or spatial dependence of the elastic or plastic effects could be included. White's work represents the first attempt to combine elastic and plastic response characteristics in a single analysis. In other words the analysis aimed at presenting a unified theory which would be valid in the regime $q / h \ll \delta$ and would go over to the rigid-plastic analysis for $q / h \sim \delta$. This work will be described later. 
Barnes, et al. (1974) conducted an experimental study in which aluminum plates were accelerated by explosive gases expanding across a void. They found significant growth for perturbation wavelengths twice the thickness of the plate at a given initial amplitude. A single additional shot (Shot 2) at half the wavelength and half the amplitude resulted in significantly less growth. The result was ascribed to a wavelength effect with the growth of the shorter wavelength perturbation being damped much more by the yield strength of the solid just as in the case of viscosity in a fluid. Numerical calculations modeling these experiments indicated a significant dependence on yield strength.

Dienes (1978), using a fully nonlinear modal technique, determined an equation of motion for a rigid-plastic fluid and found that there was a minimum acceleration required for instability. His equations in the small amplitude regime are exactly the equations of Miles in the limit $h \rightarrow \infty$. Qualitatively, the response was found to be the same for both linear and nonlinear modeling.

Drucker (1980) also obtained an equation similar to Equation 3 for $f^{\prime}$ positive. His analysis results in a $\delta$ which does not depend on the wavelength but does depend on the shape of the perturbation. The analysis does not reduce to the fluid limit as the yield strength goes to zero. Drucker (1980) also produced an additional paper detailing various non-dimensional parameters of interest in the study of Rayleigh-Taylor instability in solids.

Due to the suggestion by Drucker that the initial amplitude rather than the wavelength was the dominant factor in the no growth result for Shot 2, Barnes, et al. (1980) conducted two additional shots. The first shot essentially verified the previous result of Shot 2, and the second shot had an initial perturbation wavelength 1.86 times that of Shot 2 but at the same small amplitude. In this case, little growth was observed. It was concluded that the minimum amplitude criterion emphasized by Drucker was apparently correct.

Swegle (1987) has described the results of a set of numerical experiments conducted using a Lagrangian finite-difference wavecode to compute the time-dependent motion of elastic-plastic plates loaded by a given pressure history on an initially perturbed surface. With the exception of White's analysis all of the approximate theories discussed above are incomplete in the sense that many of the qualitative features seen in the numerical experiments cannot be reproduced. Below we shall extend and discuss White's analysis and review a second technique which was proposed earlier (Robinson and Swegle, 1987). This approach, which is distinct from White's theory, yields a coupled set of equations combining both elastic and plastic effects. A final approach is then introduced which unites various aspects of the above elastic-plastic analyses and yields a fairly simple self-consistent equation of motion. The result is strictly self-consistent only in the unstable regime in the wavelength-initial amplitude plane. The three differ- 
ent combined elastic-plastic analyses are all able to reproduce the qualitative features of the calculated solid plate response, although each approach suffers from some form of inconsistency. A major inadequacy of all the models is that, while qualitative features of the response are very favorable, the quantitative details differ significantly from the numerical plate acceleration studies. This is perhaps to be expected due to the simplicity of the assumed velocity field and the constitutive assumptions. The incompressible models are clearly incapable of providing insight into the phenomena which are due primarily to time dependent wave propagation. The models do provide, however, the best known approximate techniques for understanding the Rayleigh-Taylor response of solids. 


\section{The Modal Technique and the Constitutive Equa- tions}

We employ a one-degree-of-freedom approximation technique in that we assume that the kinematics of the flow follows the incompressible irrotational motion corresponding to the linearized stability problem for an inviscid fluid with the maximum strain amplitude, $q$, as the unknown. Thus, the velocity field is given by $\mathbf{v}=\left(v_{x}, v_{y}\right)$ where $v_{x}=\dot{q} e^{-k y} \sin k x$ and $v_{v}=\dot{q} e^{-k y} \cos k x$. We assume for simplicity that the density of the driving fluid is negligible, although the modal technique to be described can also be implemented in the case of a non-zero density driving fluid (Robinson and Swegle, 1987).

The Cauchy stress tensor for plane incompressible motion is of the form

$$
\mathbf{T}=-p \mathbf{I}+\mathbf{S}
$$

where $\mathbf{S}$ is the deviatoric part of the stress, i.e. $\mathbf{S} \cdot \mathbf{I}=0$. Since we allow only in-plane motions the components $t_{x z}$ and $t_{y z}$ of $\mathbf{T}$ are zero by symmetry. Out-of-plane strains and strain rates are zero as well. In addition, the incompressibility assumption for any of the constitutive relations which we consider will imply that the out-of-plane deviatoric stress component is zero. Thus the out-of-plane stress $t_{z z}=-p=\left(t_{x x}+t_{y v}\right) / 2$. See Hill (1950, p. 129) for a discussion in the case of a rigid-plastic material or an elastic-plastic material with Poisson's ratio $=1 / 2$. The rate-of-strain tensor, $\mathbf{D}$, is defined by

$$
\mathrm{D}=\left(\nabla \mathrm{v}+\nabla \mathbf{v}^{T}\right) / 2
$$

Incompressibility implies that $\mathbf{T} \cdot \mathbf{D}=\mathbf{S} \cdot \mathbf{D}$.

An equation for $q$ is needed and is obtained from an energy balance or power expended theorem (Gurtin, 1981, p. 110) of the form

$$
\int_{\partial V^{t}} \tilde{\mathbf{s}}(\mathbf{n}) \cdot \mathbf{v} d A+\int_{V^{t}}(\mathbf{b}-\rho \dot{\mathbf{v}}) \cdot \mathbf{v} d V=\int_{V^{t}} \mathbf{T} \cdot \mathbf{D} d V
$$

where $\tilde{\mathbf{s}}(\mathbf{n})$ is the boundary traction vector and the equivalent body force is given by $\mathbf{b}=-\rho g \hat{\mathbf{y}}$ where $\hat{\mathbf{y}}$ is the unit vector in the $y$ direction. The equivalent body force balances the boundary pressure terms so that in our frame of reference there is no zeroeth order motion. The volume $V^{t}$ depends on time. Since we restrict ourselves to plane motions, the volume integration is over an area and the surface integral becomes 
a corresponding line integral. The lower and upper surface variations, $\eta_{1}(x, t)$ and $\eta_{2}(x, t)$, respectively, of the plate must satisfy the kinematic conditions

$$
\frac{\partial \eta_{l}}{\partial t}+v_{x} \frac{\partial \eta_{l}}{\partial x}=v_{y} \text { on } y=h \delta_{2 l}+\eta_{l} \text { for } l=1,2 \text {. }
$$

The motion is assumed to be periodic in $x$ of wavelength $\lambda$ and wavenumber $k=2 \pi / \lambda$ with an imposed zero stress boundary condition on the upper surface and an imposed constant pressure on the lower surface. The volume $V^{t}$ is taken over one wavelength. One can then show, using the periodicity condition and the kinematic boundary conditions, that the boundary traction term in Equation 7 is zero. Evaluating the remaining term on the left hand side of Equation 7 to quantities of $O\left(q^{2}\right)$ in the amplitude yields

$$
-\frac{\rho \lambda \dot{q}}{2 k}\left(1-e^{-2 k h}\right)(\ddot{q}-g k q)=\int_{V t} \mathbf{S} \cdot \mathbf{D} d V .
$$

The one-degree-of-freedom modal approximation technique reduces simply to an evaluation of the right hand side of Equation 9, the stress power integral, given various assumptions about the deviatoric stress dependence on the kinematics. We now review various forms for this dependence. For an incompressible Newtonian fluid we have

$$
\mathbf{S}=2 \mu \mathbf{D}
$$

where $\mu$ is the viscosity. The Levy-Mises flow rule with the von-Mises yield criterion (rigid-plastic) has the form

$$
\mathbf{S}=\sqrt{2} s_{1} \mathbf{D} / \sqrt{\mathbf{D} \cdot \mathbf{D}}
$$

where $s_{1}$ is the von-Mises yield strength in pure shear. For an elastic-plastic incompressible material, we shall assume a Prandtl-Reuss flow rule and von-Mises yield stress criterion of the form

$$
\dot{\mathbf{S}}+\beta \mathbf{S}=2 G \mathbf{D}
$$

where $G$ is the elastic shear modulus and

$$
\beta=\frac{G \dot{W}}{s_{1}^{2}} \text { provided } J_{2}=2 s_{1}^{2} \text { and } \dot{W}>0
$$


or

$$
\beta=0 \text { provided } J_{2}<2 s_{1}^{2} \text { or } \dot{W}<0
$$

where $\dot{W}=\mathbf{S} \cdot \mathbf{D}$ and $J_{2}=\mathbf{S} \cdot \mathbf{S}$. See Hill (1950, II.5 and III.2) for a discussion of plastic flow rules. For convenience, we define the tensor $\mathbf{M}$ by

$$
\mathbf{M}=\mathbf{D} /(\dot{q} k)
$$

so that

$$
\mathbf{M} \cdot \mathbf{M}=2 e^{-2 k y} \equiv 2 c^{2} \text {. }
$$

In the inviscid fluid case, $\mathbf{S}=\mathbf{0}$, evaluation of Equation 9 yields a linear ordinary differential equation for $q$ which has the same characteristic values as the eigenvalues corresponding to the inviscid fluid Rayleigh-Taylor eigenfunction. In the case of a nonperfect fluid one must satisfy no-slip velocity and stress balance boundary conditions across an interface between two fluids. Since the inviscid fluid eigenfunction does not satisfy the correct boundary conditions, this leads to an inaccurate approximation for those modes for which the shear stress contribution is important. However, the qualitative features of the response may still be preserved using the modal technique. This will be illustrated in the cases of an incompressible Newtonian viscous fluid and an incompressible elastic solid where the exact linear stability analyses can be completed in closed form.

\subsection{Application to a Newtonian Fluid and an Elastic Solid Layer}

We can compare the above approximation technique to the exact solution when the plate is an incompressible Newtonian fluid or elastic solid layer of infinite extent. In the Newtonian fluid case, Equation 10 holds, and we have $\mathbf{S} \cdot \mathbf{D}=2 \mu k^{2} \dot{q}^{2} \mathbf{M}^{2}$. Equation 9 simplifies to

$$
\bar{q}-g k q=-4 \nu k^{2} \dot{q}
$$

where $\nu=\mu / \rho$. Assuming a solution proportional to $e^{n t}$ we obtain the characteristic equation 


$$
n^{2}-g k=-4 \nu k^{2} n \text {. }
$$

This may be compared with the characteristic equation for $n$ derived from the general two-fluid dispersion relation for $k h \rightarrow \infty$ given by Chandrasekar (1961) (taken in the limit of zero density and viscosity in the lower fluid)

$$
n^{2}-g k=-4 \nu k^{2} n+4 k^{4} \nu^{2}\left(\sqrt{1+n / k^{2} \nu}-1\right) .
$$

The last term on the right hand side of Equation 20 can be considered the contribution to the characteristic equation due to the need to satisfy velocity and stress continuity boundary conditions at the interface. One expects the approximate solution to be correct in the long wavelength limit, but wrong in the short wavelength limit since in the latter case viscosity effects should be more important. Taking leading order terms in a small $k$ expansion of Equation 20 results in Equation 19, the same equation obtained by the modal technique. The comparison of the inviscid fluid eigenfunction modal technique versus the complete Newtonian fluid solution problem was first made by Miles and Dienes (1966). They determined the maximum growth rate to be $0.40\left(g^{2} / \nu\right)^{1 / 3}$ for the approximate analysis and $0.46\left(g^{2} / \nu\right)^{1 / 3}$ for the exact analysis. Figure 3 illustrates the growth rate curves as a function of wavenumber for the exact solution and for the approximations discussed above. Both the magnitude of the growth rate and the wavenumber of maximum growth rate are displaced from the exact values. The example indicates that the inviscid fluid eigenfunction approximation technique can perform quantitatively well in the long wavelength limit, but may provide qualitatively correct results over the full wavelength range.

A similar analysis can be easily obtained for the case of a perfectly elastic incompressible fluid. Recall that we assume a factor $e^{\text {nt }}$ for the time dependence of all quantities. Thus, examining Equation 12 with $\beta=0$ leads to a correspondence between $G / n$ and the $\mu$ of the Newtonian analysis. The dispersion relation in the elastic case will be obtained by replacing $\mu$ in Equation 19 by $G / n$. One obtains the exact relation

$$
n^{2}-g k=-4(G / \rho) k^{2}+4 k^{4}(G /(\rho n))^{2}\left(\sqrt{1+n^{2} \rho /\left(k^{2} G\right)}-1\right) .
$$

The long wavelength limit of this equation and the modal technique both yield

$$
n^{2}=g k-4 G k^{2} / \rho .
$$

See Figure 4. The scaling on the plot emphasizes the weaknesses of the long wavelength approximation due to the fact that the cutoff wavelength is not correctly predicted. 
However, the comparison does indicate that replacing the constant $G$ in the long wavelength analysis by one half its nominal value may provide the best agreement with a more accurate analysis in so far as the cutoff wavelength and wavelength of maximum growth rate are concerned. This would, however, increase the maximum growth rate by a factor of two.

\subsection{Rigid-Plastic Equations}

As an extended case of the rigid-plastic model equations represented by Equation 2 , it is instructive to determine the modal stability equation for the case of a yield strength which depends upon pressure. We assume that the yield strength $\mathrm{Y}$ varies linearly across the plate with the pressure. Thus for constant $d Y / d p=\alpha$ we obtain

$$
Y=Y_{h}+\alpha p_{0}(h-y) / h
$$

where $Y_{h}$ is the nominal yield strength of the unloaded material on the back surface of the plate. The analysis follows as usual by evaluating Equation 9 with $S$ given by Equation 11. The important detail is that $\mathbf{S} \cdot \mathbf{D}$ has a more complicated dependence on $y$, and the stress power integration takes this into account. Recalling that $s_{1}=Y / \sqrt{3}$, one obtains

$$
\dot{q}-g k q=-\frac{4 k \operatorname{sgn} \dot{q}}{\sqrt{3} \rho\left(1+e^{-k h}\right)}\left\{Y_{h}+\alpha p_{0}\left(\frac{1}{1-e^{-k h}}-\frac{1}{k h}\right)\right\} .
$$

This is exactly Equation 2 except for an additional dependence of the right hand side on $k h$ which represents an additional contribution for $d Y / d p$ non-zero. It is easy to show that the following inequalities hold for all $\alpha p_{0} \geq 0$ and $k h \geq 0$ :

$$
Y_{h} \leq Y_{h}+\alpha p_{0}\left\{\frac{1}{1-e^{-k h}}-\frac{1}{k h}\right\} \leq Y_{h}+\alpha p_{0}
$$

The term in braces takes on a value of .5 at $k h=0$ and approaches 1.0 monotonically as $k h$ increases. Thus one finds, as expected, that the stabilizing effect of the linearly varying yield strength is bounded by that seen if the whole plate were composed of a material characterized by the least and the maximum yield strength of the linearly varying plate. This is consistent with numerical calculations for which the yield strength depends on pressure (Robinson and Swegle, 1987; Swegle, 1987). 


\section{Elastic-Plastic Modal Analyses}

One of the principal qualitative characteristics for Rayleigh-Taylor instability response in solids found numerically (Robinson and Swegle, 1987; Swegle, 1987) is the existence of a boundary in amplitude-wavelength space separating solutions which become unbounded (unstable) from those which oscillate and remain bounded (stable). This boundary is strongly dependent on wavelength in the sense that for large enough wavelengths all initial amplitudes are unstable. For wavelengths smaller than a certain cutoff wavelength only sufficiently small initial amplitudes will be stable. These characteristics cannot be obtained by any of the modeling equations discussed so far. A combined analysis wherein both the elastic shear modulus and the yield strength play a part is required. Three different approaches to this type of unified theory are discussed below.

The first approach (labeled E-P-I) is similar to the work of White (1973) but in the end results in a a simpler and more consistent analysis. White showed how one could solve the Prandtl-Reuss stress rate equation for $\mathbf{S} \cdot \mathbf{D}$ exactly, assuming that the flow was on the yield surface. His analysis was inconsistent in that it did not allow for a purely elastic response in the stress power integration and made ad hoc assumptions about the appropriate region of integration for the stress power integral. We point out how different assumptions about the initial deviatoric stress affect the analysis and choose an assumption which appears to be the most advantageous. The second theory (E-P-II) takes a finite element approach to a representation of the deviatoric stress to derive a coupled set of equations for the perturbation growth and a stress parameter $s$. The theory takes into account regions of elastic and plastic response, but must allow for plastic deviatoric stress states which violate the von-Mises yield criterion in order to carry out the analysis. This theory was first presented by Robinson and Swegle (1987). A final theory (E-P-III) attempts to combine the best features of E-P-I and E-P-II so that a properly consistent account of elastic and plastic regions is made without sacrificing the standard definition of the yield criterion as is done with both E-P-I and E-P-II. However, E-P-III is weak in the sense that bookkeeping difficulties preclude a fully consistent solution for stable solution trajectories.

The small amplitude assumption which we have employed for the stress power integral has been clear when the deviatoric stress is proportional to the strain or strain rate as in the sample calculations of Section 2.1. The result is that the stress power integral can be computed over the undeformed configuration without regard to surface variation. In Section 2.2 we implicitly used this same assumption, and we shall continue to do so. This is acceptable since we are interested in time scales given by $1 / \sqrt{g k}$ and we may employ the small amplitude balance $q / h \sim s_{1} / p_{0} \ll 1$ such as discussed in the introduction. However, $G k q / s_{1}$ is not assumed to be small. 


\section{$3.1 \quad$ E-P-I}

White (1973) assumed that all material in the plate would be on the yield surface and stay on the yield surface so that Equations 12 and 13 would hold throughout the flow. We rederive his results in our notation and make a few additional comments and extensions. Taking the inner product of Equation 12 with $\mathbf{M}$ and defining

$$
\begin{aligned}
\bar{x} & =\mathbf{S} \cdot \mathbf{M} /\left(2 s_{1} c\right) \\
& =\left(\left(t_{x x}-t_{y y}\right) /\left(2 s_{1}\right)\right) \cos k x-\left(t_{x y} / s_{1}\right) \sin k x \\
& =a_{1} \cos k x+a_{2} \sin k x
\end{aligned}
$$

and

$$
\eta=2 G k q / s_{1}
$$

one obtains

$$
\frac{d \bar{x}}{d \eta}+c \bar{x}^{2}=c
$$

This is a Ricatti equation which may be solved by standard techniques to yield

$$
\bar{x}=\frac{\tanh \left[c\left(\eta-\eta_{0}\right)\right]+\bar{x}\left(\eta_{0}\right)}{1+\bar{x}\left(\eta_{0}\right) \tanh \left[c\left(\eta-\eta_{0}\right)\right]}
$$

where $\eta_{0}=2 G k q(0) / s_{1}$. Now by the Cauchy-Schwarz inequality and the yield condition, $|\mathbf{S} \cdot \mathbf{M}| \leq|\mathbf{S}||\mathbf{M}| \leq\left(\sqrt{2} s_{1}\right)(\sqrt{2} c)=2 s_{1} c$ so that $\left|\bar{x}\left(\eta_{0}\right)\right| \leq 1$. The small amplitude assumption we have employed implies that we may neglect surface variations in the stress power integral of Equation 9. Therefore, we may perform either the $x$ or the $y$ integration first in the evaluation of the double integral. Assuming that the $a_{i}$ are independent of $y$ and integrating with respect to $y$, we obtain

$$
\begin{aligned}
\int_{0}^{h} \mathbf{S} \cdot \mathbf{D} d y & =\int_{0}^{h} 2 s_{1} k \dot{q} c \frac{\sinh \left[c\left(\eta-\eta_{0}\right)+\tanh ^{-1}\left(\bar{x}\left(\eta_{0}\right)\right)\right]}{\cosh \left[c\left(\eta-\eta_{0}\right)+\tanh ^{-1}\left(\bar{x}\left(\eta_{0}\right)\right)\right]} d y \\
& =\frac{2 s_{1} \dot{q}}{\left(\eta-\eta_{0}\right)} \log \left\{\frac{\cosh \left[\left(\eta-\eta_{0}\right)+\tanh ^{-1}\left(\bar{x}\left(\eta_{0}\right)\right)\right]}{\cosh \left[e^{-k h}\left(\eta-\eta_{0}\right)+\tanh ^{-1}\left(\bar{x}\left(\eta_{0}\right)\right)\right]}\right\} .
\end{aligned}
$$


The integration with respect to $x$ depends on the assumed form of $\bar{x}\left(\eta_{0}\right)$ and explicit assumptions about the form of $\bar{x}\left(\eta_{0}\right)$ must be made. If the $a_{i}$ are assumed independent of $x$ then by expanding the cosh functions, simplifying and using classical results from complex variable theory, we can integrate Equation 33 over a full wavelength and substitute in Equation 9 to obtain

$$
\begin{gathered}
\bar{q}-g k q=-\frac{4 s_{1} k}{\rho\left(\eta-\eta_{0}\right)\left(1-e^{-2 k h}\right)} \times \\
\log \left\{\frac{\cosh \left(\eta-\eta_{0}\right)+\sqrt{1+\left(1-a^{2}\right) \sinh ^{2}\left(\eta-\eta_{0}\right)}}{\cosh \left(e^{-k h}\left(\eta-\eta_{0}\right)\right)+\sqrt{1+\left(1-a^{2}\right) \sinh ^{2}\left(e^{-k h}\left(\eta-\eta_{0}\right)\right.}}\right\}
\end{gathered}
$$

where $a=a_{1}^{2}+a_{2}^{2}$.

White proposed the initial condition $\bar{x}\left(\eta_{0}\right)=a_{1} \cos k x$ so that $a_{2}=0$. He noted, however, that this assumption is not always consistent with a positive stress power as can be seen by evaluating Equation 31 for small values of the argument $\eta-\eta_{0}$. We now look at two limiting cases of Equation 34. For small $\eta-\eta_{0}$, Equation 34 yields

$$
\bar{q}-g k q=-2\left(2-a^{2}\right) G k^{2}(q-q(0)) / \rho .
$$

White claimed that integration over only a half wavelength (so that the leading coefficient on the right hand side of Equation 35 would be replaced by unity) would match the small amplitude results of Miles if $a_{1}=\sqrt{3} / 2$ which corresponds to Miles' assumptions on the initial stress state. This claim does not appear to be correct. In the case of large $\eta-\eta_{0}$, Equation 34 leads directly to Equation 2 so that the rigid-plastic response equation is obtained.

In the incompressible plane strain approximation, it would seem in general to be more appropriate to assume either zero initial deviatoric stress, or a deviatoric stress which is consistent with the assumed strains. In the case of zero initial deviatoric stress, $a=0$ and Equation 34 becomes

$$
\tilde{q}-g k q=-\frac{4 s_{1} k}{\rho\left(\eta-\eta_{0}\right)\left(1-e^{-2 k h}\right)} \log \left\{\frac{\cosh \left(\eta-\eta_{0}\right)}{\cosh \left(e^{-k h}\left(\eta-\eta_{0}\right)\right)}\right\}
$$

Equation 36 is the result we call E-P-I and was not discussed by White. Examination of the properties of the equation leads to the conclusion that it may be a good candidate for a full elastic-plastic theory. If we consider motions for which $q(0)=0$, and look at small 
$\eta$, then Equation 34 with $a=0$ becomes the exact elastic stability equation in the long wavelength limit (Equation 21). For large $\eta$ one obtains Miles' rigid-plastic Equation 2. The question immediately arises as to why we get the correct small amplitude response when the derivation assumed that the flow would be on the yield surface. The explanation is that the $\bar{x}^{2}$ term in Equation 29 does not enter at leading order for the initial condition $\bar{x}\left(\eta_{0}\right)=0$ so that a small $\eta-\eta_{0}$ expansion gives essentially an elastic response equation at leading order. Despite its origins, Equation 35 thus appears to be a good candidate for an approximate global elastic-plastic stability response equation since it has the correct limiting response in both the small amplitude elastic and large amplitude fully plastic regimes.

The second alternative assumption on the initial stress state, which is consistent with an incompressible plane strain approximation, is to take an initial deviatoric stress state which is colinear with the assumed deviatoric strain yet lies on the yield surface. In this case $\bar{x}\left(\eta_{0}\right)=1$, and from Equation 30 we see that $\bar{x}(\eta)=1$. Integration of the stress power then leads to Equation 2, the rigid plastic stability equation.

\section{$3.2 \quad$ E-P-II}

The energy integral approach can be thought of in terms of a one-term Galerkin approximation. A natural way to handle the stress rate Equation 12 is then to assume a specific form for the stress deviator $\mathbf{S}$, multiply the equation by an appropriate test function and then integrate over the appropriate volume. Following this line of reasoning, we assume that $\mathbf{S}=s \mathbf{M}$ and use $\mathbf{M}$ itself as the test function. Thus we obtain

$$
\int_{V^{t}} \dot{\mathbf{S}} \cdot \mathbf{M} d V+\int_{V^{t}} \beta \mathbf{S} \cdot \mathbf{M} d V=\int_{V^{t}} 2 G \mathbf{D} \cdot \mathbf{M} d V .
$$

The second integral on the left hand side need not be taken over the full volume, but only over those regions in which $\beta$ is non-zero. Due to the simple form of our basis function, we must allow for deviatoric stress states outside the yield surface so that now the definition of the parameter $\beta$ of Equations 13 and 14 becomes

$$
\begin{aligned}
\beta & =\frac{G \dot{W}}{s_{1}^{2}} \text { provided } J_{2} \geq 2 s_{1}^{2} \text { and } \dot{W}>0 \\
& =0 \text { provided } J_{2}<2 s_{1}^{2} \text { or } \dot{W}<0
\end{aligned}
$$

Therefore, the region of $y$ integration in the second integral is bounded by the solution to the equation 


$$
J_{2}=2 s_{1}^{2}=s^{2} M^{2}=2 s^{2} c^{2}=2 s^{2} e^{-2 k y} .
$$

That is, we must integrate over the region $0 \leq y \leq \min (\max (0, \hat{y}), h)=\bar{y}$ where

$$
\hat{y}=-\frac{1}{2 k} \log \left(s_{1} / s\right)^{2} .
$$

Evaluating Equation 36 we obtain the equation for the evolution of $s$ as

$$
\dot{s}+H(s \dot{q}) G k \dot{q}\left(s / s_{1}\right)^{2} \frac{\int_{0}^{\lambda} \int_{0}^{0} \mathbf{M}^{4} d y d x}{\int_{0}^{\lambda} \int_{0}^{h} \mathbf{M}^{2} d y d x}=2 G k \dot{q}
$$

where $H$ is the Heaviside function. It should be recognized that the second term in Equation 41 is formally $O\left(\dot{q} s^{2}\right)$. However, since $s_{1}^{2}$ appears in the denominator as well and we consider motions for which $s / s_{1}$ is bounded, we may include this term without loss of consistency. (We shall see that $s / s_{1} \lesssim 1.55$ for initial conditions of interest). This second term represents the only nonlinear aspect of the analysis and leads to the amplitude effects to be discussed later. Using the definitions for $\hat{y}$ and $\bar{y}$ and $\mathbf{S}=s \mathbf{M}$, we then obtain the coupled set of equations, which we call E-P-II, by evaluating Equations 9 and 41

$$
\begin{gathered}
\dot{q}-g k q=-2 k s / \rho \\
\dot{s}=2 G k \dot{q} \times\left\{\begin{array}{ll}
1 & \text { for }|\mathrm{s}| \leq \mathrm{s}_{1} \text { or } \mathrm{s} \dot{\mathrm{q}}<0 \\
1-\left(\left(s / s_{1}\right)^{2}-\left(s / s_{1}\right)^{-2}\right) /(2 \gamma) & \text { for } \mathrm{s}_{1} \leq|\mathrm{s}| \leq \mathrm{s}^{*} \text { and } \mathrm{s} \dot{\mathrm{q}}>0
\end{array}\right\}
\end{gathered}
$$

where $s^{*}=s_{1} \sqrt{\gamma+\sqrt{\gamma^{2}+1}}$ and $\gamma=1-e^{-2 k h}$. Note that $\dot{s}$ equals zero when $s=s^{*}$.

We may now look at various limits of the above equations in order to compare with the equations derived by Miles. For $|s|<s_{1}$, the nonlinear term in the stress rate equation is absent and thus the general solution of Equations 42 and 43 for constant $g$ is

$$
\left(\begin{array}{c}
q \\
\dot{q} \\
s
\end{array}\right)=c_{1}\left(\begin{array}{c}
2 k / \rho \\
0 \\
g k
\end{array}\right)+c_{2}\left(\begin{array}{c}
1 \\
n \\
2 G k
\end{array}\right) e^{n t}+c_{3}\left(\begin{array}{c}
1 \\
-n \\
2 G k
\end{array}\right) e^{-n t}
$$


where $\mathrm{n}=\sqrt{g k-4 G k^{2} / \rho}$ and the $\mathrm{c}_{i}$ are constants. For initial conditions, $\dot{q}(0)=0$, $s(0)=0$, the solution is

$$
\left(\begin{array}{c}
q \\
\dot{q} \\
s
\end{array}\right)=\frac{q(0)}{n^{2}}\left(\begin{array}{c}
g k \cosh (n t)-4 G k^{2} / \rho \\
g k n \sinh (n t) \\
2 G g k^{2}(\cosh (n t)-1)
\end{array}\right) \text {. }
$$

The dominant exponentially growing terms in the above solution satisfy

$$
\bar{q}-\left(g k-\frac{4 G k^{2}}{\rho}\right) q=0
$$

which is our usual elastic stability equation. For $s=s^{*}$, we have $\dot{s}=0$ and we obtain

$$
\ddot{q}-g k q=-\frac{2 k s^{*}}{\rho}
$$

which may be compared with Equation 2 derived by Miles. The equivalent value of $s^{*} / s_{1}$ for Miles' solution and the $s^{*} / s_{1}$ for the E-P-II analysis are shown in Figure 5 . Respectively, the asymptotic limits as $k h \rightarrow \infty$ are 2 and $\sqrt{1+\sqrt{2}} \simeq 1.55$.

\subsection{E-P-III}

The development of E-P-II in the previous section is convenient and straightforward in approach but is weak in the sense that the yield criteria had to be modified prior to integration over the test volume. It is appealing, however, because both elastic and plastic regions are included in the analysis and a proper self-consistent accounting can be made within the framework of the approximations. In contrast, the approach leading to E-P-I solves the equations of motion exactly, assuming the flow is on the yield surface everywhere, but does not precisely allow for regions of the flow where one should assume either an elastic or a plastic response.

A theory which does provide a unification of the best features of the previous two approaches will be outlined below. We develop a very simple equation of motion which is properly self-consistent for unstable trajectories. However, we are unable to follow through with the analysis for oscillatory stable solutions due to bookkeeping difficulties arising when a fully consistent solution would require the creation of a multiplicity of 
elastic and plastic regions. However, the final result is instructive and clearly shows the transition from elastic to plastic behavior.

Our third technique satisfies Equations 13 and 14 precisely in the evaluation of the quantity $\mathbf{S} \cdot \mathbf{M}$. Recall that we define $\mathbf{M}=\mathbf{D} / \dot{q} k$ and $\mathbf{M}^{2}=2 c^{2}=2 e^{-2 k y}$. Taking the inner product of Equation 12 with $D$ and $S$, when Equation 14 holds, leads to differential equations for $\mathbf{S} \cdot \mathbf{M}$ and $\mathbf{S}^{\mathbf{2}}$, respectively, which may be solved successively to obtain

$$
\begin{gathered}
\mathbf{S} \cdot \mathbf{M}=4 G k c^{2}(q-q(0)) \\
\mathbf{S}^{2}=8 G^{2} k^{2} c^{2}(q-q(0))^{2}=(\mathbf{S} \cdot \mathbf{M})^{2} /\left(2 c^{2}\right)
\end{gathered}
$$

where here we assume that $\mathbf{S}(0)=0$. Setting $q(0)=0$ is equivalent to assuming initial elastic and plastic strains corresponding to motion from infinitesimal strains.

The equation $\mathbf{S}^{2}=2 s_{1}^{2}$ and Equation 49 define a boundary between elastic response in which Equation 48 should be used in the stress power integral and plastic response in which another solution for $\mathbf{S} \cdot \mathbf{M}$ must be derived. To derive this solution, we multiply $M$ into Equation 12, with $\beta$ given by Equation 13, to obtain the differential equation

$$
\frac{d}{d t}(\mathbf{S} \cdot \mathbf{M})+G \dot{q} k(\mathbf{S} \cdot \mathbf{M})^{2} / s_{1}^{2}=4 G \dot{q} k c^{2}
$$

with an initial condition given by the value of $\mathbf{S} \cdot \mathbf{M}$ when $\mathbf{S}^{2}=2 s_{1}^{2}$. From Equation 49 this value is determined to be $\mathbf{S} \cdot \mathbf{M}=2 s_{1}$ c. But this constant also satisfies Equation 50 so that $\mathbf{S} \cdot \mathbf{M}$ in the plastic region is exactly $2 s_{1} c$ ! Equation 50 is, in fact, Equation 29 in the E-P-I analysis, but in the present case the solution can be determined by inspection. In contrast to E-P-I the approach taken here gives results for a consistent time-dependent formulation of the stress power integral in which elastic and plastic behaviors are exactly determined. We can evaluate the stress power integral using the correct amplitude dependent form of $\mathbf{S} \cdot \mathbf{M}$ for the elastic and plastic regions. Recalling the definition of $\eta$ in Equation 28, this computation gives the following equation valid for unstable trajectories $\left(\tilde{\eta}=\eta-\eta_{0} \geq 0\right.$ and $\left.\dot{q}>0\right)$ :

$$
\bar{q}-g k q=-2 s_{1} k f(\tilde{\eta}) / \rho
$$

where 


$$
f(\tilde{\eta})=\left\{\begin{array}{cc}
\tilde{\eta} & 0 \leq \tilde{\eta} \leq 1 \\
\frac{2-\tilde{\eta}^{-1}-\tilde{\eta} e^{-2 k h}}{1-e^{-2 k h}} & 1 \leq \tilde{\eta} \leq e^{k h} \\
\frac{2}{1+e^{-k h}} & \tilde{\eta}>e^{k h}
\end{array}\right.
$$

The $\tilde{\eta}<1$ limit of Equation 51 for $q(0)=0$ is clearly our usual long wavelength stability equation. Equation 51 goes through a transition phase for $\tilde{\eta}>1$ and for $\tilde{\eta}>e^{k h}$ becomes identically the usual rigid-plastic equation. Although we do not elaborate here, the restriction $\dot{q}>0$ can be relaxed somewhat, and the solution can be consistently extended for small oscillatory deviations from a constant amplitude. However, the bookkeeping difficulties of keeping track of elastic and plastic regions soon become overwhelming for large amplitude oscillations. As with the previous analyses, however, we can ignore the derivational inconsistency and use the above results directly for negative as well as positive $\dot{q}$. Equation 51, with the restriction on $\dot{q}$ lifted, is the result we term E-P-III. 


\subsection{Solution Behavior}

The elastic-plastic stability equations derived in the previous three sections can be integrated numerically for a variety of initial conditions and parameter choices. The equations of interest are Equation 35 (E-P-I), the set of coupled Equations 42 and 43 (EP-II) and Equation 51 (E-P-III). For purposes of this paper, we show mainly results from integration of the E-P-II equations. The nominal parameter values for an aluminum plate and the initial conditions as implemented in the computations are $\dot{q}(0)=0$, $s(0)=0, G=15 \mathrm{GPa}, Y=.325 \mathrm{GPa}, \rho=2700 \mathrm{~kg} / \mathrm{m}^{3}$ and $h=2.54 \mathrm{~mm}$. The value $G$ has been taken to be roughly half the nominal value in order to better approximate the $\lambda_{m}$ of the exact solution for a perfectly elastic plate (Robinson and Swegle, 1987). The pressure profile for the results to be presented below (excluding Figure 9) consists of a linear ramp over $1.4 \mu$ s to a maximum constant pressure $p_{m}$. The initial amplitude $q(0)$, the perturbation wavelength $\lambda$ and $p_{m}$ will be varied parametrically.

Figure 6 represents a typical numerical solution of Equations 42 and 43 . For small initial amplitudes, the solutions are stable and the amplitude oscillates, while for a large enough amplitude, the solution is clearly unstable. When the quantity $s$ reaches the limiting value, $s^{*}$, shown by the dotted line, the solution grows very quickly, while if $s$ never reaches $s^{*}$, the solution will not grow indefinitely. In other words, when the damping effect of the stress power term no longer increases with perturbation amplitude, then the perturbation tends to grow as an inertially dominated instability. The stress power term in each of the elastic-plastic models which we have developed acts in essentially the same way. A stability boundary may also exist between stable short wavelengths and unstable long wavelengths as indicated in Figure 7. As this boundary is approached from below, the oscillations increase in period and amplitude. One also observes that in the unstable regime there is a wavelength for which the solution reaches a given amplitude first, although asymptotically the shortest wavelength will eventually have the largest growth rate. A stability boundary in initial amplitudewavelength space will be observed which depends significantly on the driving pressure as illustrated in Figure 8. A solution is classified as unstable if $q / h$ reaches 1 or $s=s^{*}$. At fairly low maximum pressures, when $G / p_{m}$ is not small, the stability boundary is insensitive to perturbation wavelength. At high maximum pressures $G / p_{m}$ is not large and the boundary is observed to be strongly dependent on wavelength. A distinct cutoff wavelength stability boundary behavior is expected and observed at $\lambda / h=8 \pi G / p_{m}=\pi$ where $p_{m}=120 \mathrm{GPa}$.

The qualitative similarity between the diagrams shown here and the detailed numerical results of Swegle (1987) is striking. The degree of quantitative agreement is, however, somewhat disappointing particularly in terms of the overestimation of the amplitude of the stability boundary. However, this is not too surprising considering the 
nature of the approximations involved and the differences in the constitutive modeling. We show in Figure 9 a comparison between the experimental results of Barnes, et al. $(1974,1980)$ and the predictions of the model equations using the nominal parameters given above and an approximate actual profile for $p_{0}$ as given by Swegle (1987, Figure 19). The overprediction of the stability boundary is typical. Also, note the expected proportional dependence of the stability boundary on the value of the yield stress for small wavelengths. When $G$ is greatly decreased, one is able to observe a dip in the stability boundary. This occurs since the longer wavelength perturbations which would have been unstable under a sustained pressure do not grow fast enough during the finite duration pulse to be classified as unstable.

A comparison of the solution characteristics for the three basic approximate techniques is made in Figures 10 and 11 for a stable oscillatory case and an unstable case. All three techniques give results which are in essential agreement. Only very small variations, which make no significant qualitative or quantitative difference, are observed. 


\section{Discussion}

The numerical experiments of Swegle (1987) and the elastic-plastic analytical approximations discussed here are in substantial qualitative agreement. The basic point of agreement is that both elasticity and plasticity may play an important role in determining the location of the stability-instability boundary in an initial amplitude-wavelength stability diagram. The major discrepancy is that the approximate modal techniques tend to greatly overestimate the critical amplitude relative to the numerical simulations. This significant quantitative error appears to be due in part to the simple form of the velocity field and the linearizing assumptions adopted in the analysis, but more importantly to the fact that the model equations do not take into account the true compressibility of the plate. Compressibility would allow for the development of plastic strains other than simple plane shear. The numerical experiments also indicate a substantial dependence of the response on the time variation of the driving pressure. This is due to the presence of pressure gradients which drive instabilities at a rate which varies markedly from the quasi-steady state pressure gradient assumption implicit in the incompressible analysis. It is felt that any more refined approximate analysis must include additional factors related primarily to compressibility effects.

In many practical cases $G / p_{0}$ is a large number and thus imposed perturbations which have a length scale on the order of the plate thickness will not grow unless a certain amplitude criterion is satisfied. Thus, in this case an initial perturbation amplitude stability criterion can be a useful indicator of stability. However, if the pressure is large enough, the stabilizing influence of the elastic shear strength will be lost for wavelengths on the order of the plate thickness. The only effect of the yield strength will then be to retard the growth of perturbations, and thus in this sense, one would not expect to be able to drive a coherent plate no matter how small the initial perturbation. 


\section{References}

1. J. F. Barnes, P. J. Blewett, R. G. McQueen, K. A. Meyer, and D. Venable, "Taylor Instability in Solids," J. Appl. Phys., 45 (2), 1974, pp. 727-732.

2. J. F. Barnes, D. H. Janney, R. K. London, K. A. Meyer and D. H. Sharp, "Further Experimentation on Taylor Instability in Solids," J. Appl. Physics, 51 (9), 1980, pp. 4678-4679.

3. S. Chandrasekhar, "Hydrodynamic and Hydromagnetic Stability," Dover Publications, Inc., New York, 1981, pp. 441-443.

4. J. K. Dienes, "Method of Generalized Coordinates and an Application to RayleighTaylor Instability," Phys. Fluids 21(5), May 1978, pp. 736-744.

5. D. C. Drucker, "Taylor Instability of the Surface of an Elastic-Plastic Plate," Mechanics Today, Vol. 5, S. Nemmat-Nasser, Ed., 1980, pp. 37-47.

6. D. C. Drucker, "A Further Look at Rayleigh-Taylor and Other Surface Instabilities in Solids," Ingenieur-Archiv 49, (1980), pp. 361-367.

7. M. E. Gurtin, An Introduction to Continuum Mechanics, Academic Press, 1981.

8. R. Hill, The Mathematical Theory of Plasticity, Oxford Univ. Press, 1950.

9. J. W. Miles, “Taylor Instability of a Flat Plate,” General Dynamics GAMD-7335, AD643161, 1966.

10. J. W. Miles and J. K. Dienes, "Taylor Instability in a Viscous Liquid," Phys. of Fluids, 9 (12), 1966, pp. 2518-2519.

11. A. C. Robinson and J. W. Swegle, "A Numerical and Analytical Investigation of Rayleigh-Taylor Instability in a Solid Tungsten Plate," Sandia National Laboratories, SAND87-0766, 1987.

12. D. H. Sharp, "An Overview of Rayleigh-Taylor Instability," Physica 12D, 1984, pp. 3-18.

13. J. W. Swegle, "A Numerical Study of Rayleigh-Taylor Instability in Accelerated Plates," Sandia National Laboratories, SAND87-2519, 1987.

14. G. N. White, "A One-Degree-of-Freedom Model for the Taylor Instability of an Ideally Plastic Metal Plate," Los Alamos National Laboratory, LA-5225-MS, Informal Report, April 1973. 


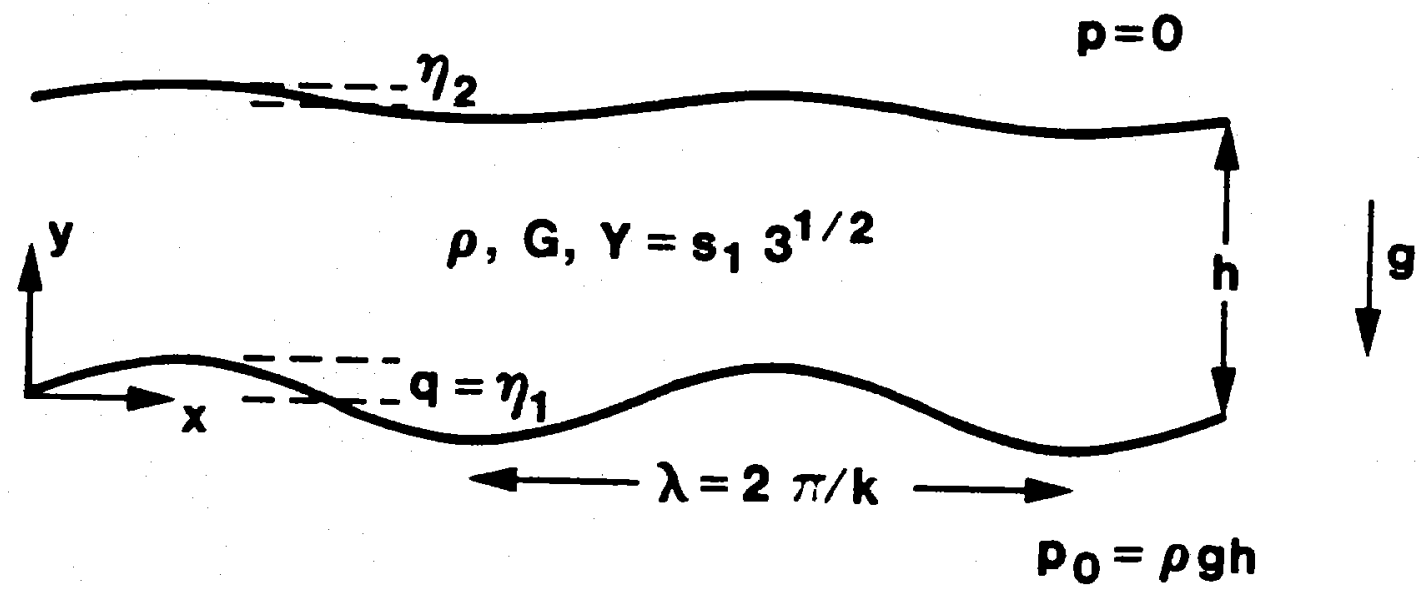

Figure 1: Rayleigh-Taylor instability of an elastic-plastic plate. 


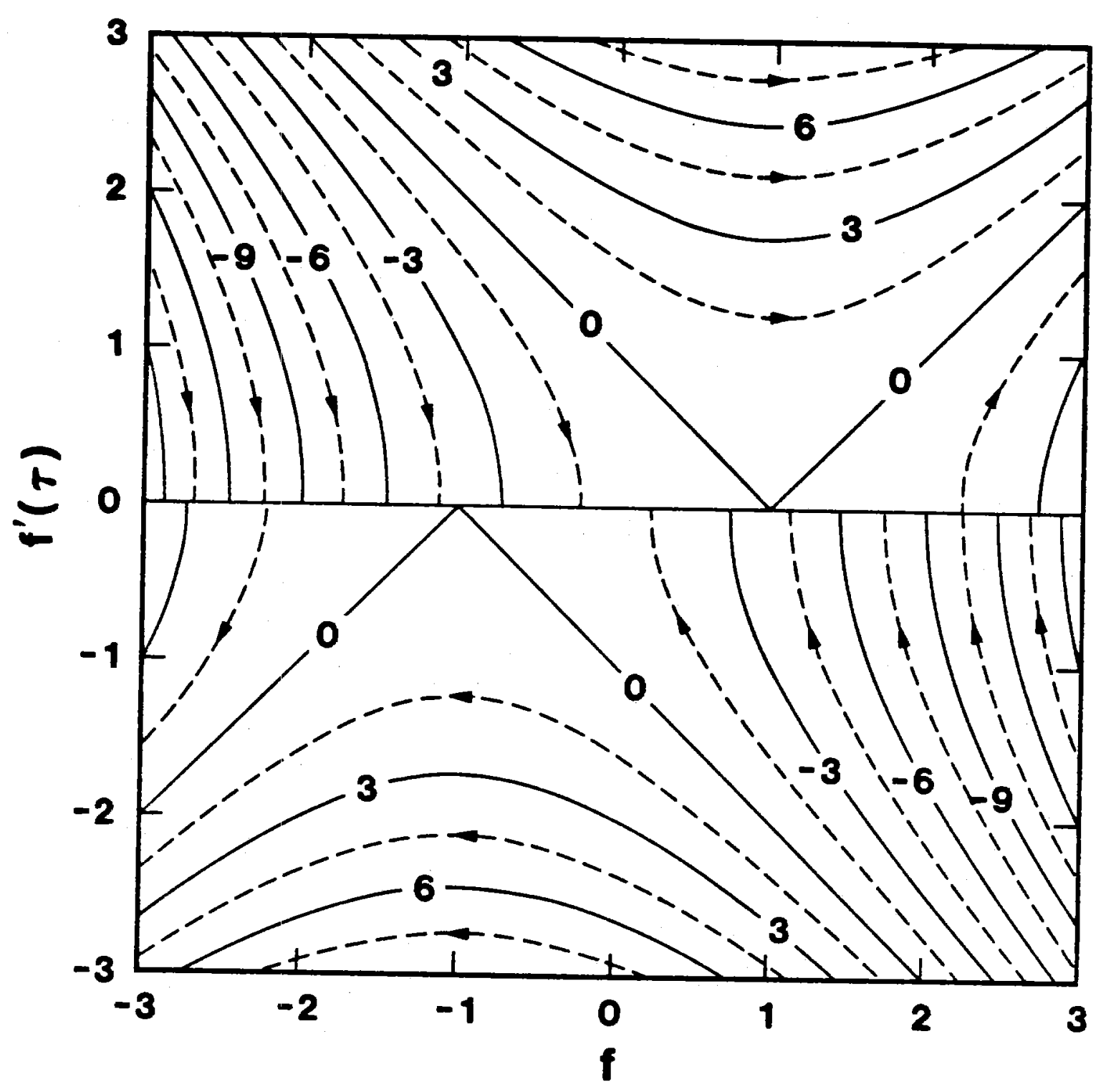

Figure 2: Phase plane for the rigid-plastic one-degree-of-freedom equation. 


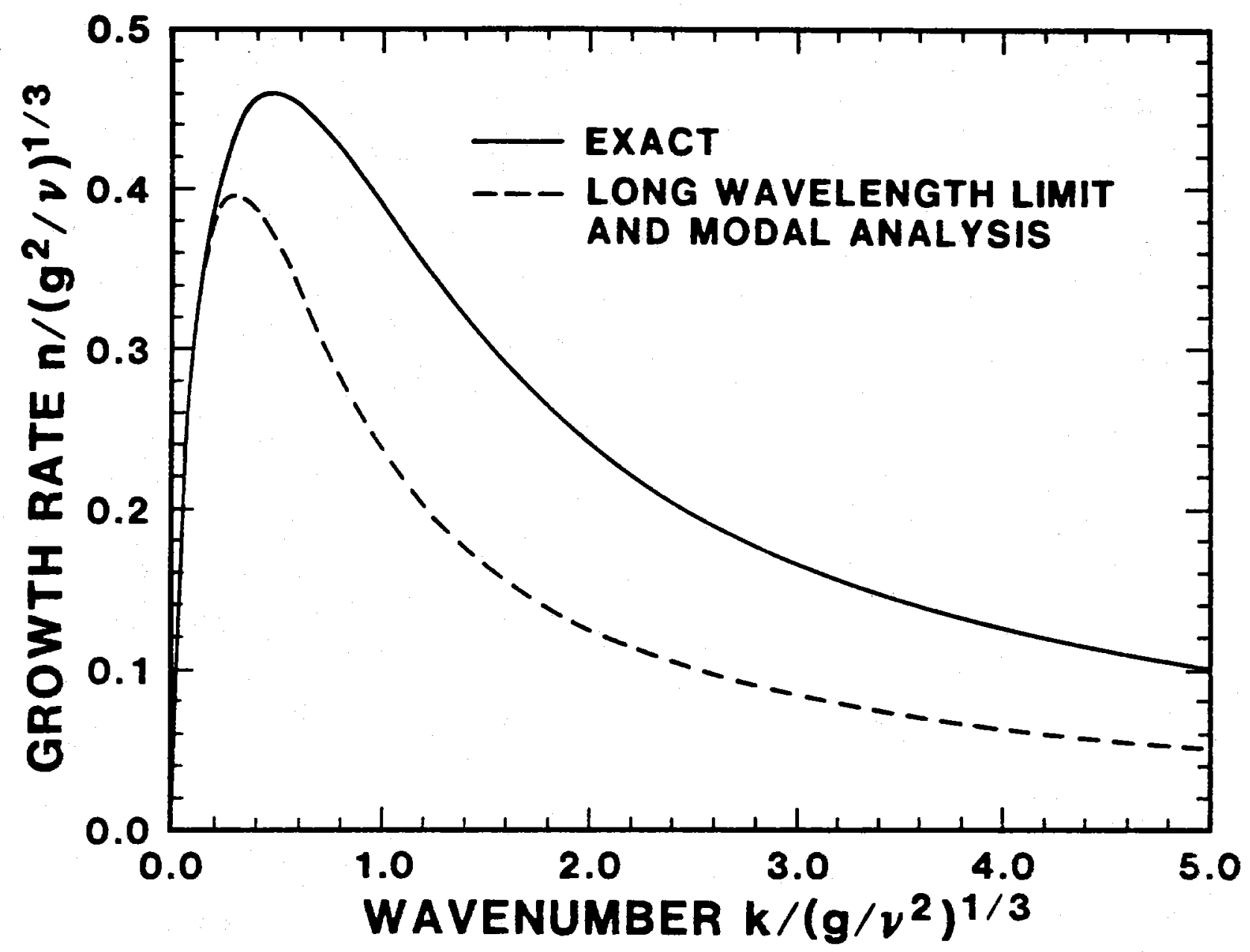

Figure 3: Growth rate comparison between the modal approximation and the exact solution for a Newtonian fluid. 


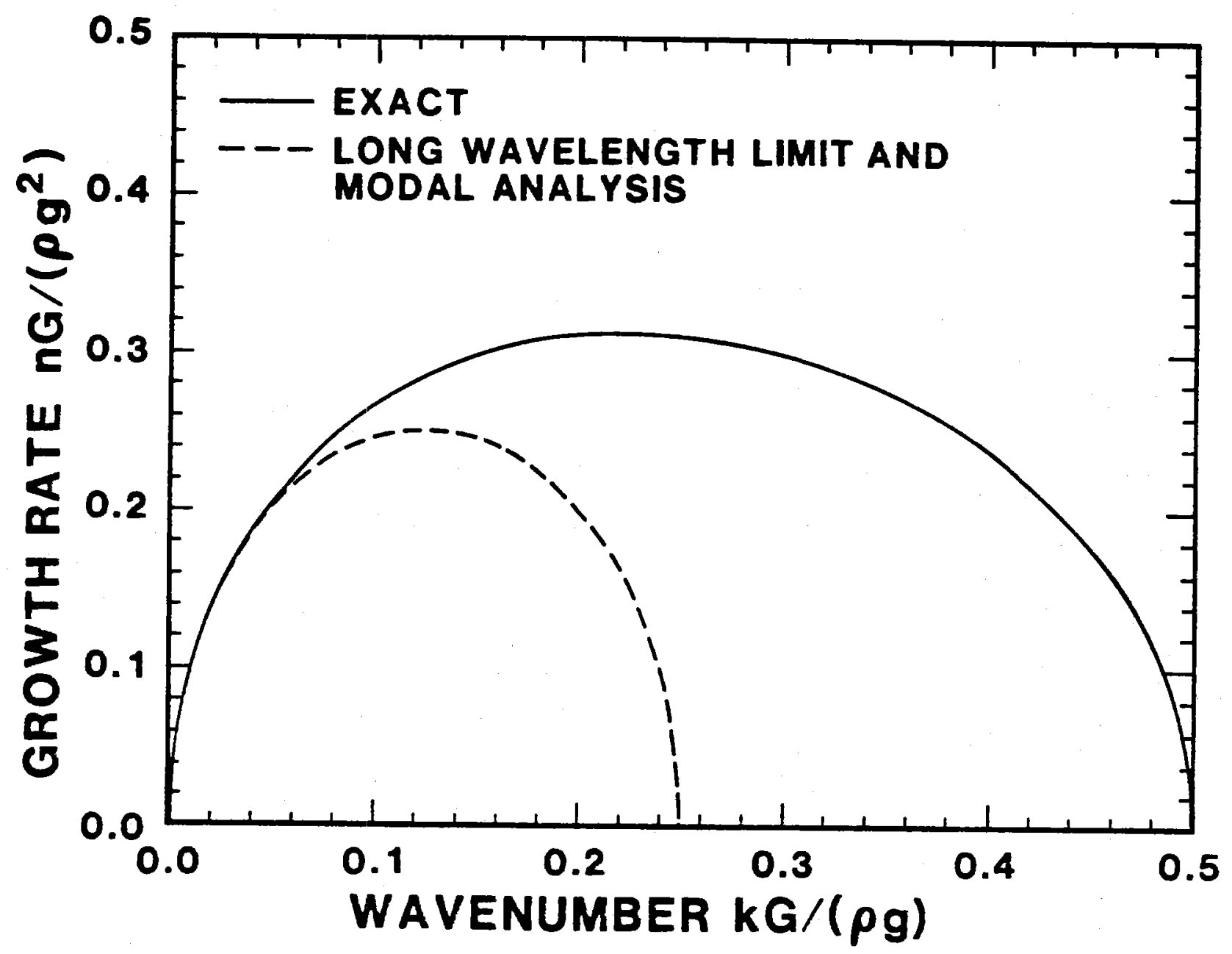

Figure 4: Growth rate comparison between the modal approximation and the exact solution for an elastic fluid. 


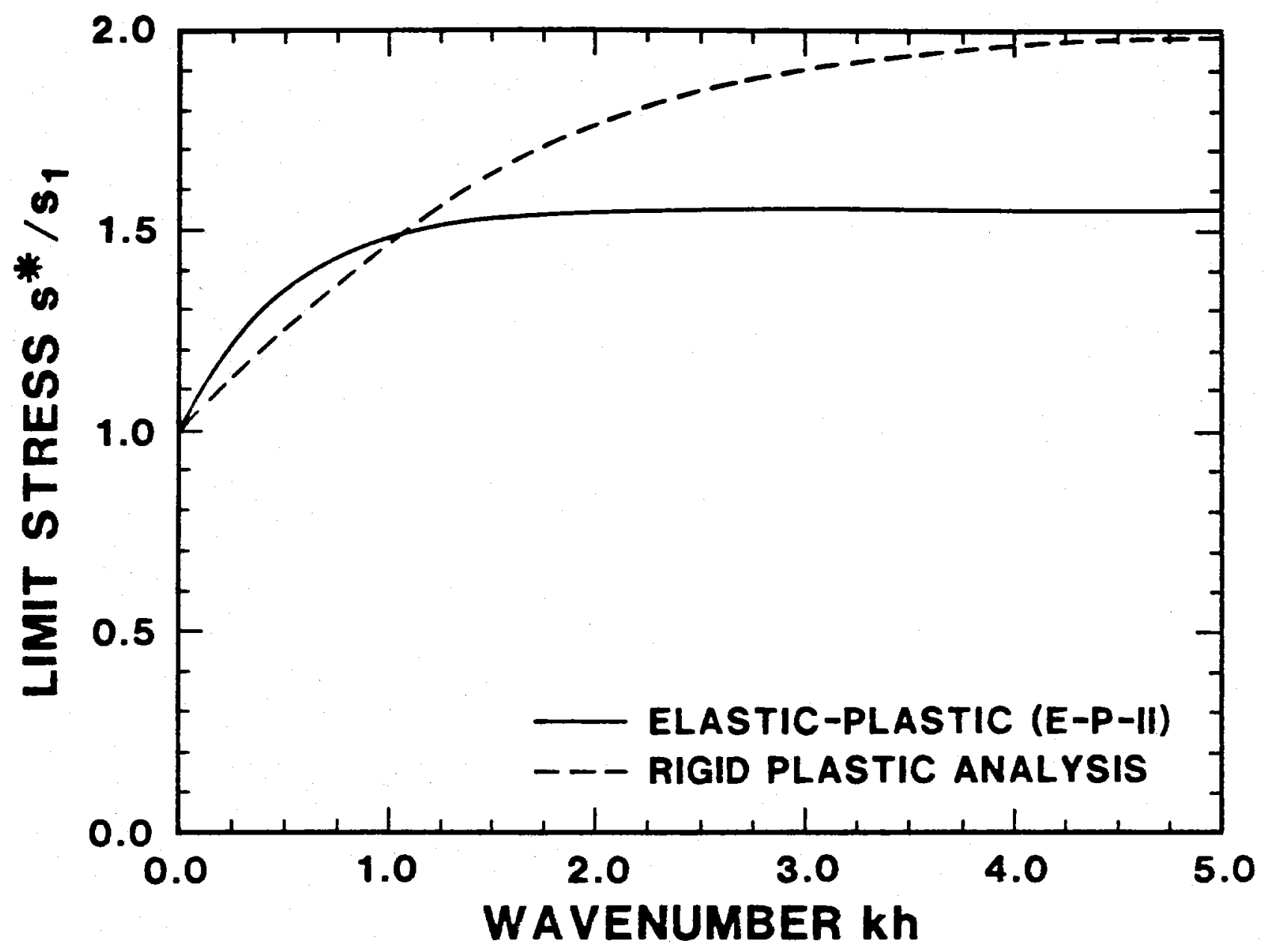

Figure 5: Limit stress comparison between the E-P-II model and the rigid plastic model. 


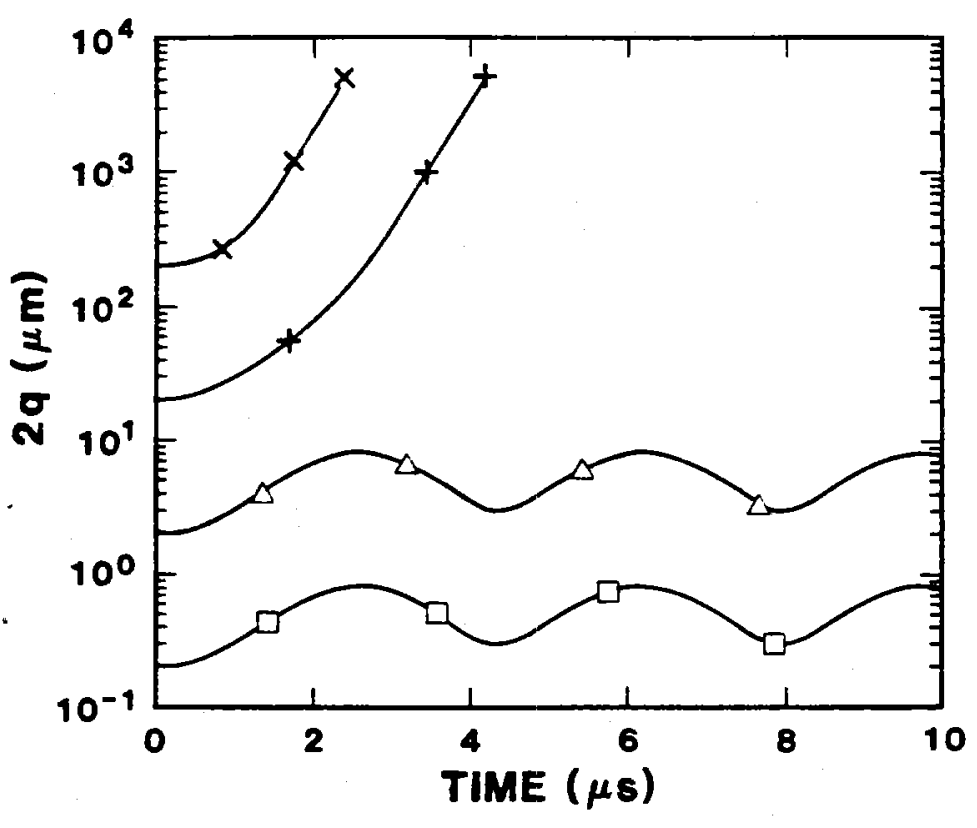

(a)

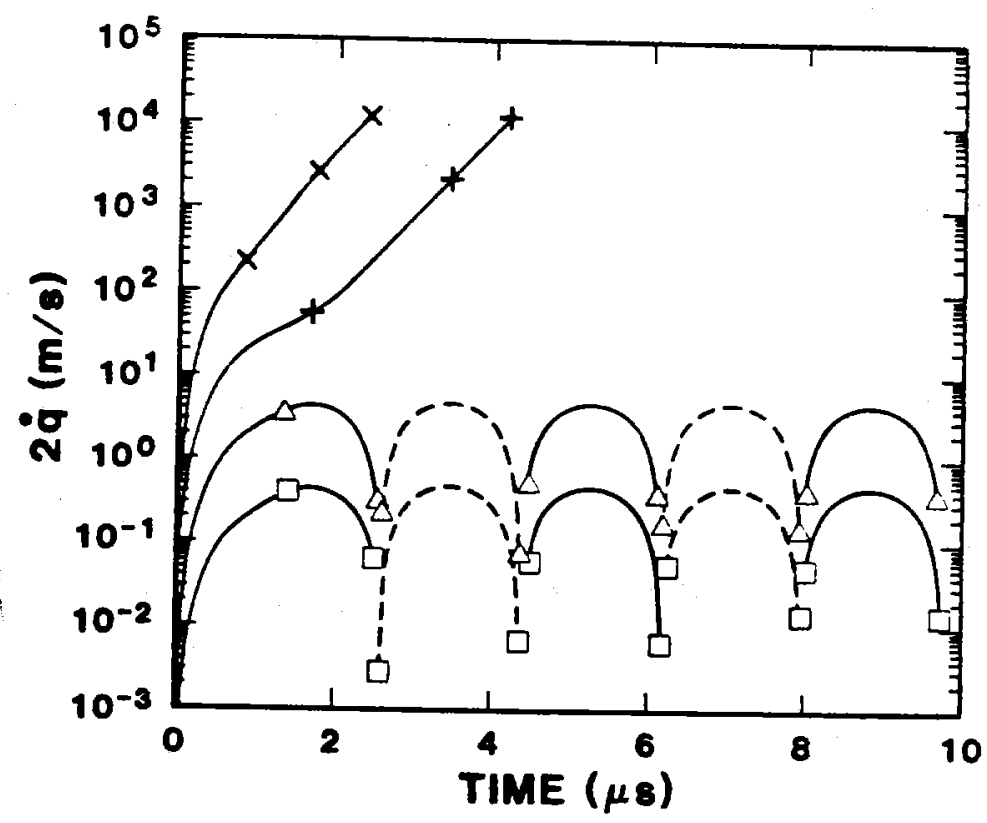

(b)

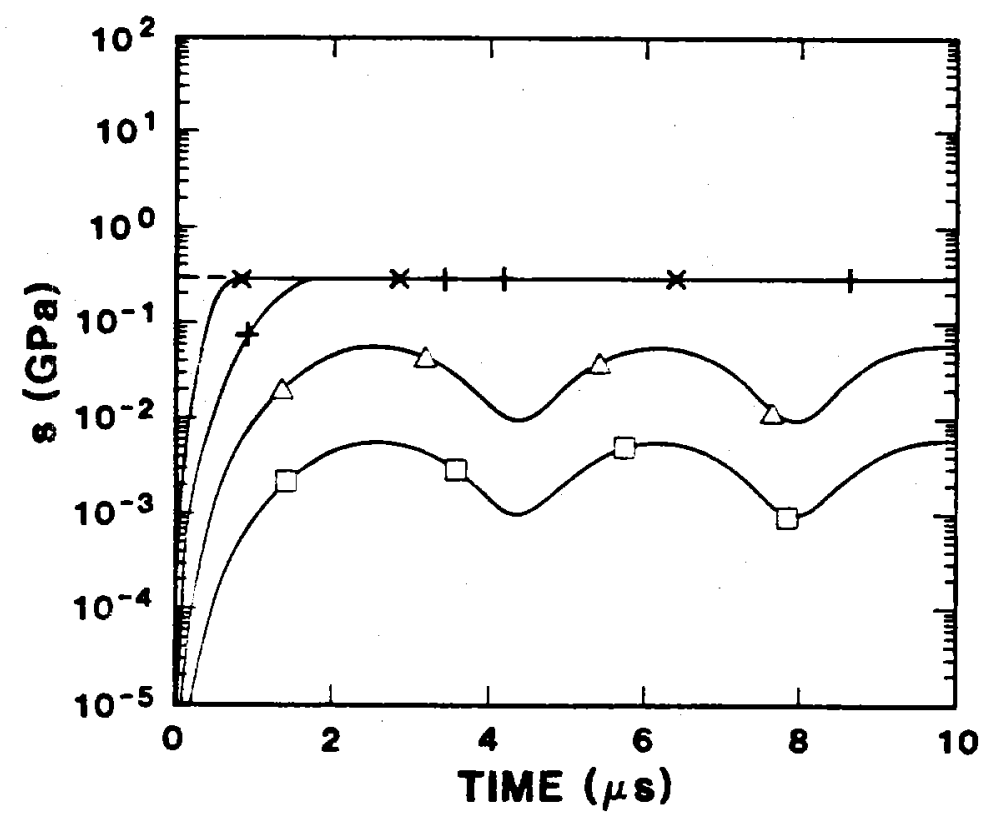

(c)

Figure 6: Amplitude $q$, velocity $\dot{q}$ and stress variable $s$ (a, b and c resp.) for $\lambda / h=4$ and $p_{m}=60 \mathrm{GPa}$ for E-P-II with different values of initial amplitude as shown in (a). Dotted lines represent negative values. 


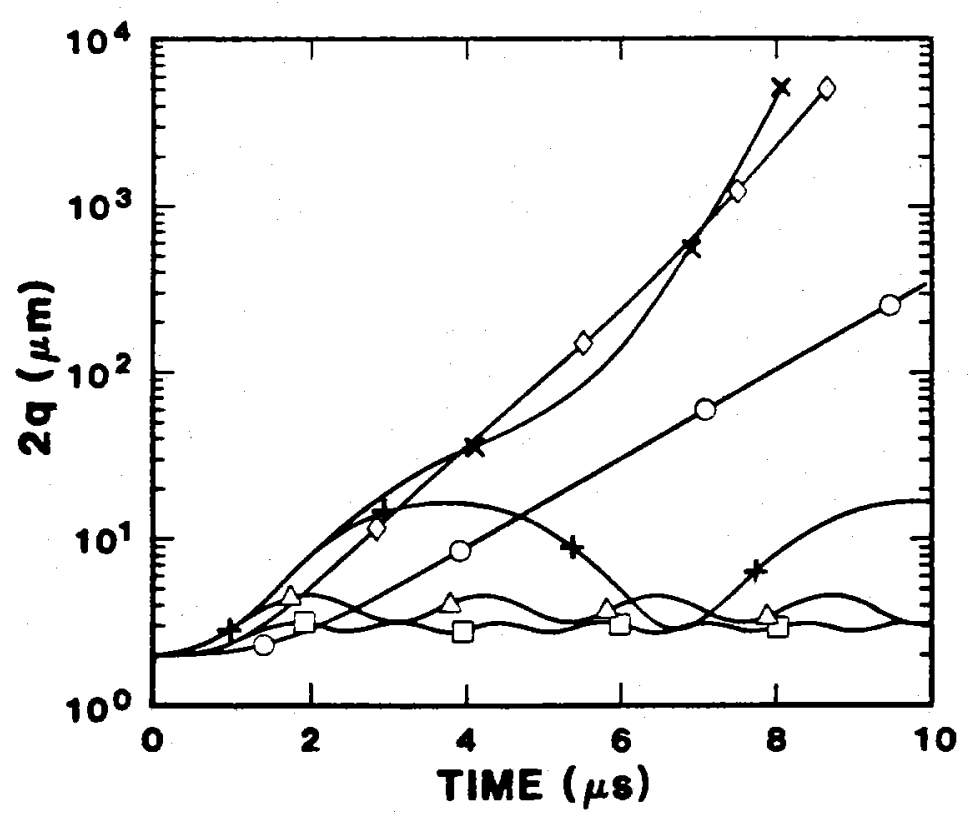

(a)

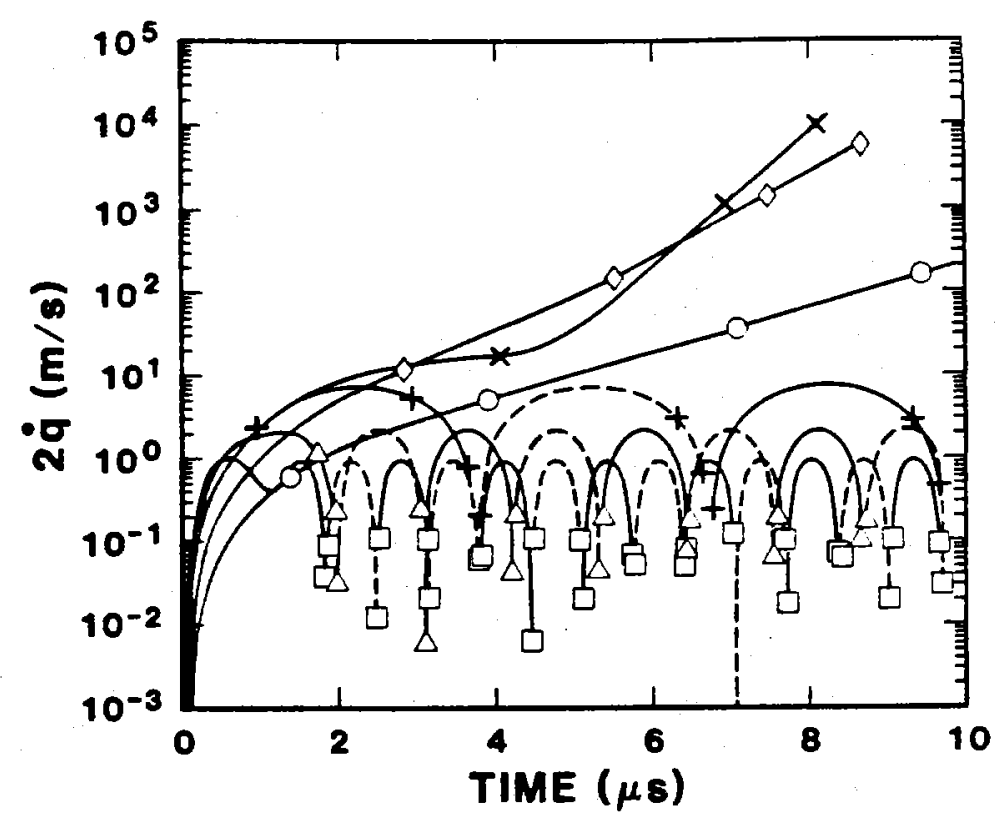

(b)

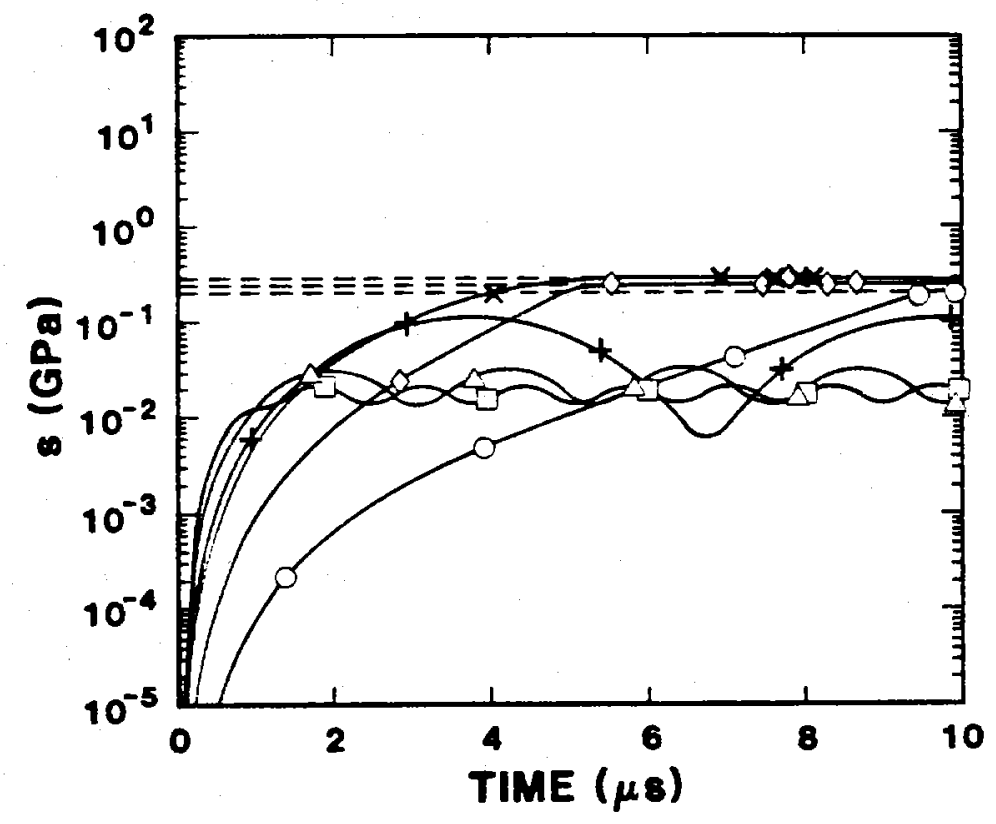

(c)

Figure 7: Amplitude $q$, velocity $\dot{q}$ and stress variable $s$ (a, b and c resp.) for $\lambda / h=2$ $(\square), 3(\triangle), 5(+), 6(\times), 15(\circ)$ and $50(\circ)$ for $p_{m}=60 \mathrm{GPa}$ and E-P-II. Dotted lines represent negative values. 


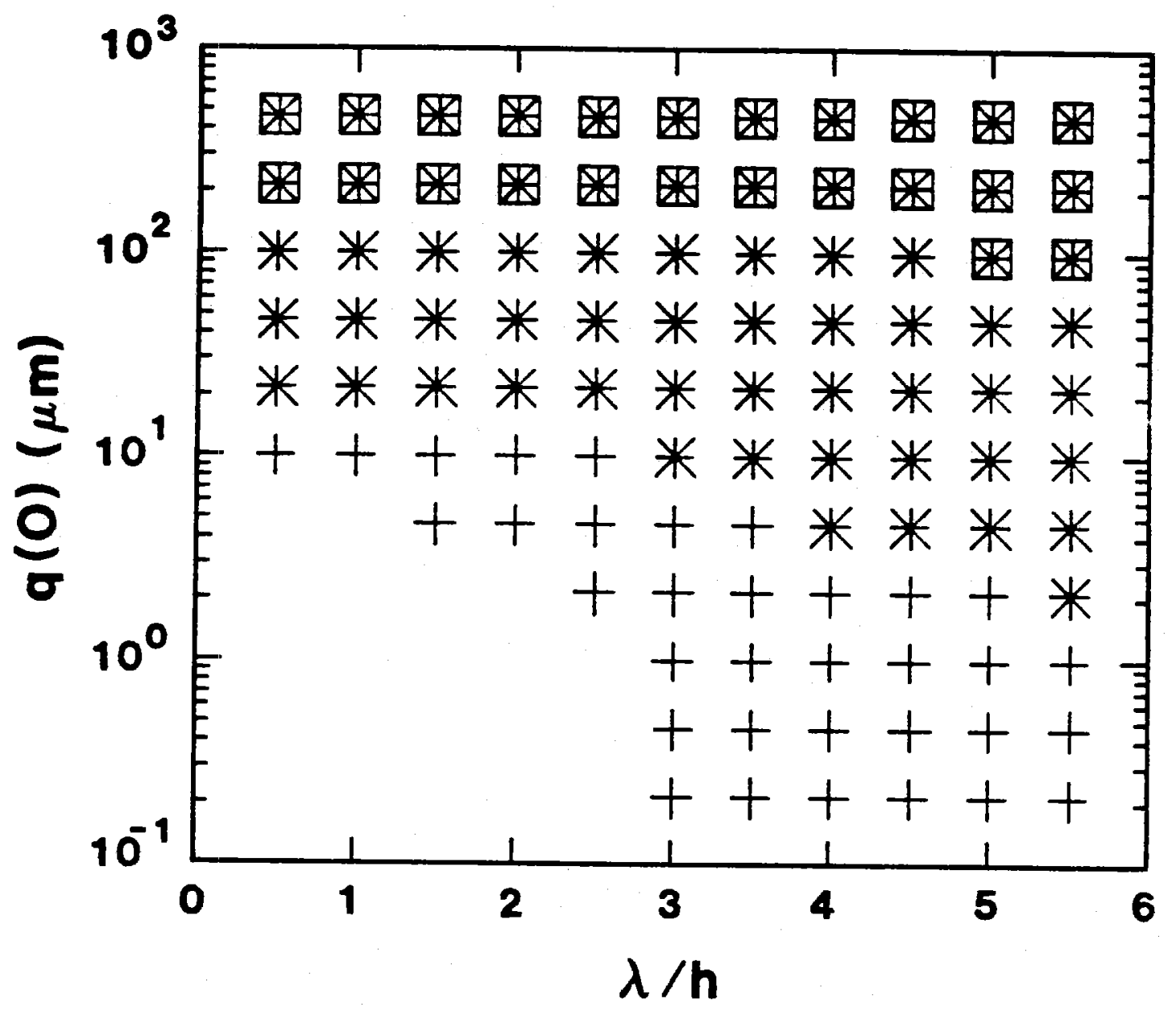

Figure 8: E-P- $\Pi$ unstable solutions for $p_{m}=10 \mathrm{GPa}(\square), 60 \mathrm{GPa}(\times)$ and $120 \mathrm{GPa}$ $(+)$. Each point represents three distinct calculations. 


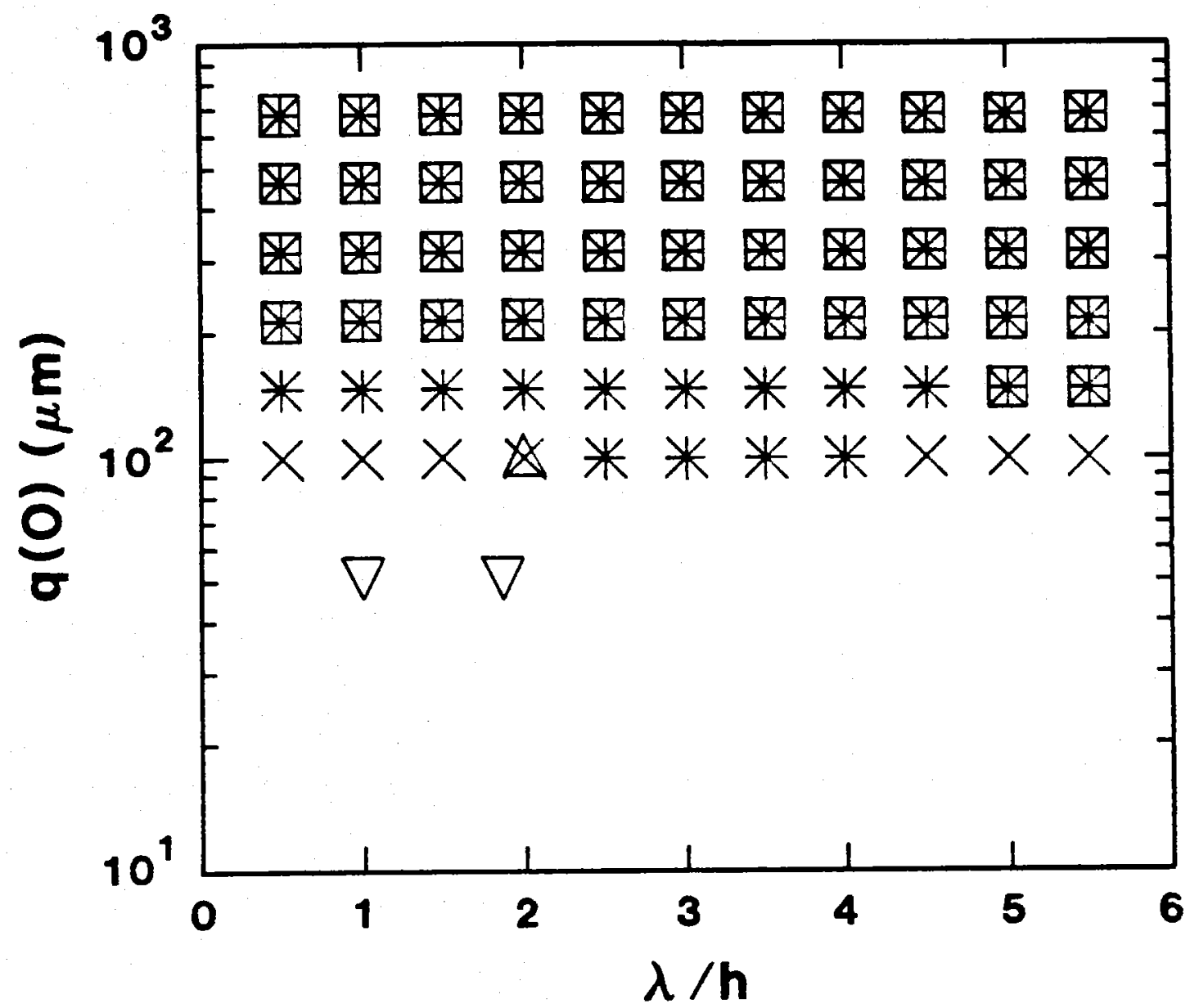

Figure 9: Comparison between E-P-II model predictions for unstable parameter points $(\square)$ and the data of Barnes, et al.: unstable $(\triangle)$ and stable $(\nabla)$. Also shown are results for $G=3 \mathrm{GPa}(+)$ and $Y=.1625 \mathrm{GPa}(\times) . G=15 \mathrm{GPa}$ and $Y=.325 \mathrm{GPa}$ for the $\square$ points. 


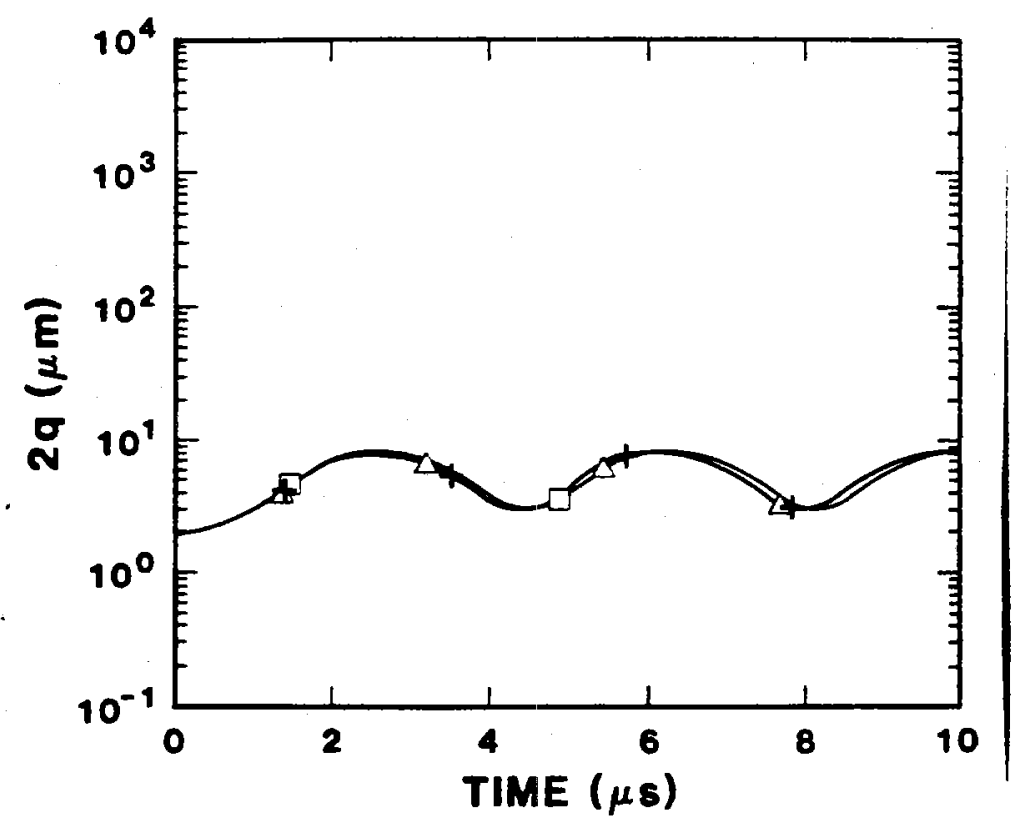

(a)

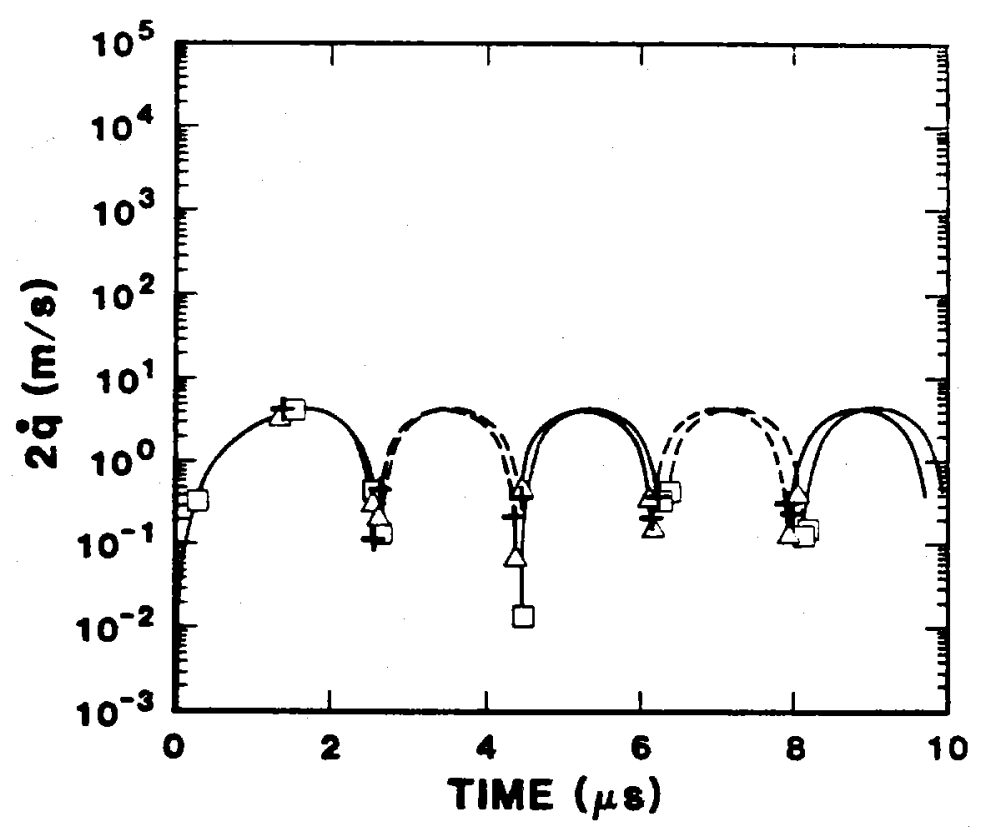

(b)

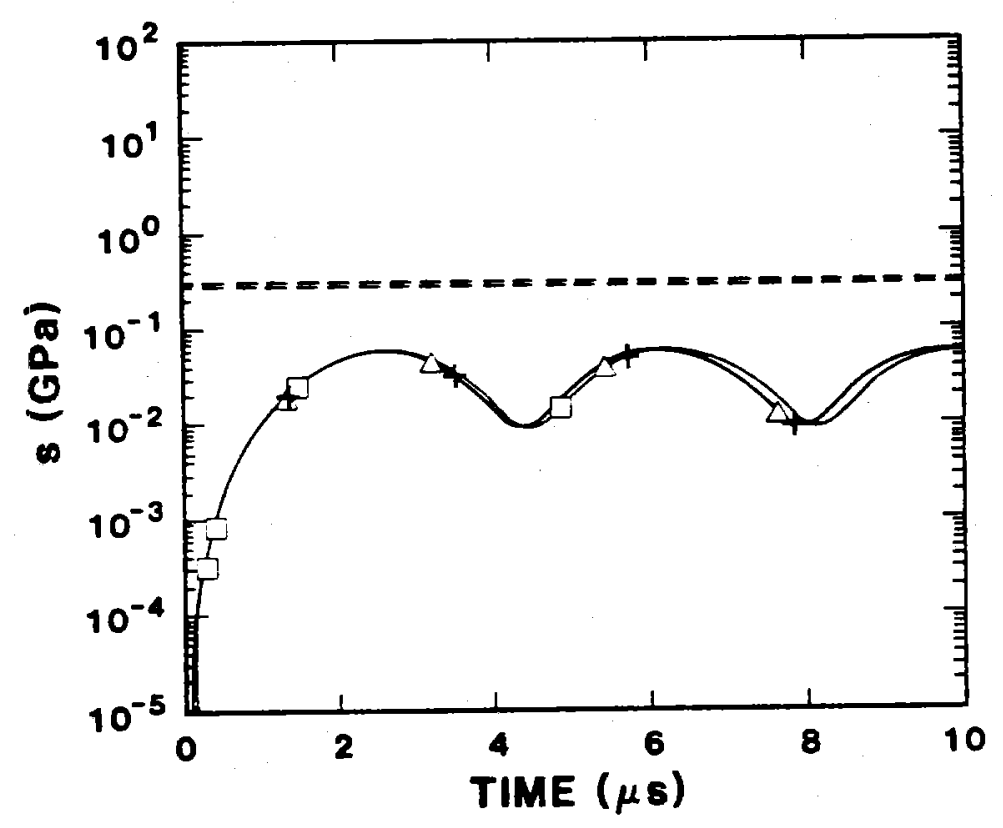

(c)

Figure 10: Amplitude $q$, velocity $\dot{q}$ and equivalent stress variables $s$ (a, b and c resp.) for E-P-I $(\square)$, E-P-II $(\triangle)$, and E-P-III $(+)$ where $q(0)=1 \mu m, \lambda / h=4, p_{m}=60 \mathrm{GPa}$ leads to an oscillatory response. Dotted lines represent negative values. 


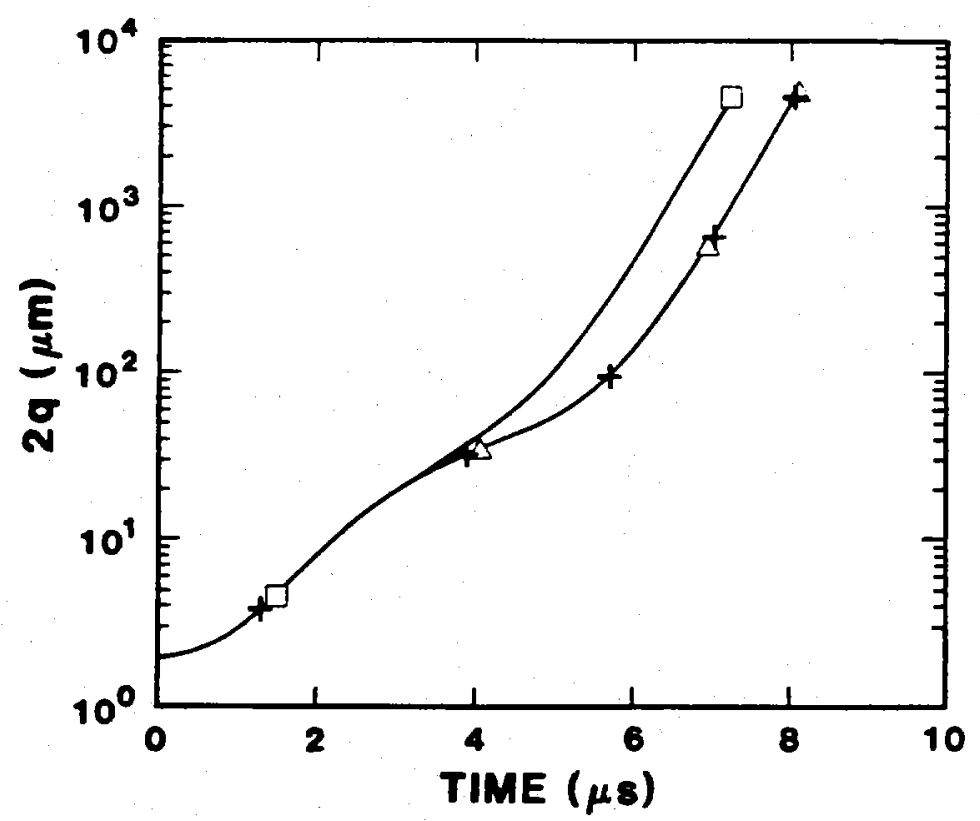

(a)

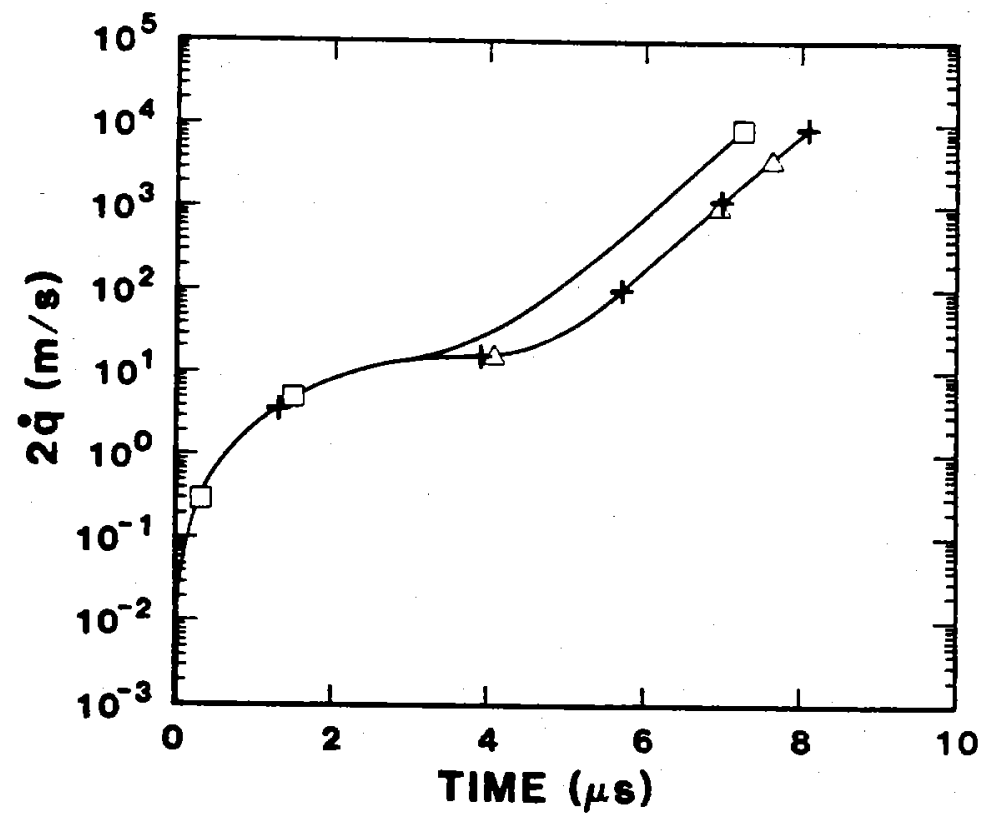

(b)

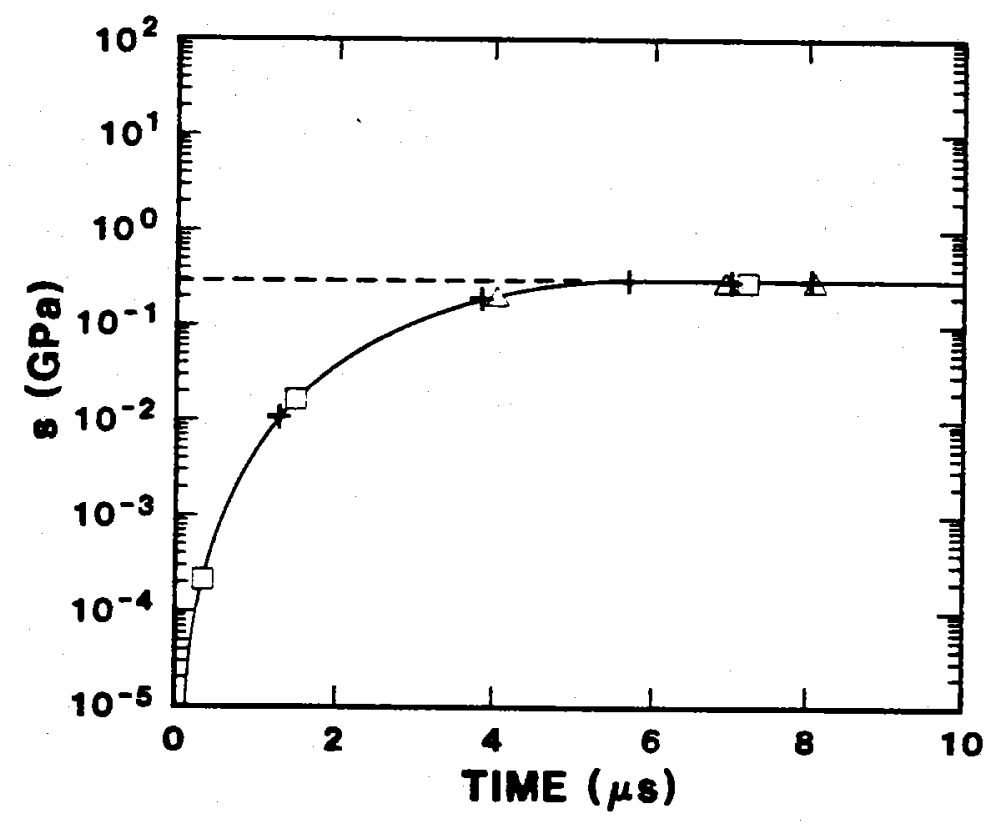

(c)

Figure 11: Amplitude $q$, velocity $\dot{q}$ and equivalent stress variables $s$ (a, b and c resp.) for E-P-I ( $\square)$, E-P-II $(\triangle)$, and E-P-III $(+)$ where $q(0)=1 \mu m, \lambda / h=6, p_{m}=60 \mathrm{GPa}$ leads to an unstable response. Dotted lines represent negative values. 


\section{DISTRIBUTION:}

J. F. Barnes, T-1, MS B221

Los Alamos National Laboratory P. O. Box 1663

Los Alamos, NM 87545

P. Blewett, X-5, MS F669

Los Alamos National Laboratory

P. O. Box 1663

Los Alamos, NM 87545

B. Daly, T-3, MS B216

Los Alamos National Laboratory

P. O. Box 1663

Los Alamos, NM 87545

J. Dienes, T-3, MS B216

Los Alamos National Laboratory

P. O. Box 1663

Los Alamos, NM 87545

S. W. Eisenhawer, Q-6, MS K557

Los Alamos National Laboratory

P. O. box 1663

Los Alamos, NM 87545

R. P. Godwin, X-3, MS F669

Los Alamos National Laboratory

P. O. Box 1663

Los Alamos, NM 87545

F. Harlow, T-3, MS B216

Los Alamos National Laboratory

P. O. Box 1663

Los Alamos, NM 87545

N. Johnson, T-3, MS B216

Los Alamos National Laboratory

P. O. Box 1663

Los Alamos, NM 87545

J. W. Miles

IGPP, A-025

University of California

La Jolla, CA 92093
T. A. Sandford, DRA/NDR, MS F617

Los Alamos National Laboratory

P. O. Box 1663

Los Alamos, NM 87545

G. R. Spillman, DRA/NDR, MS F617

Los Alamos National Laboratory

P. O. Box 1663

Los Alamos, NM 87545

J. W. Taylor, ADDRA, MS A110

Los Alamos National Laboratory

P. O. Box 1663

Los Alamos, NM 87545

Dr. M. Scharff

Science Applications, Inc.

10401 Rosella Street

San Diego, CA 92121

Dr. J. Kamm

Science Applications, Inc.

10401 Rosella Street

San Diego, CA 92121

Dr. Yasuyuki Horie

North Carolina State University

Box 7908

Rayleigh, NC 27695

\section{Sandia Internal}

1200 J. P. VanDevender

1261 T. W. Hussey

1261 S. S. Payne

1263 F. C. Perry

1422 L. A. Romero

1500 W. Herrmann

1510 J. W. Nunziato

1511 D. K. Gartling

1511 R. Givler

1512 J. C. Cummings

1512 J. R. Torczynski

1513 D. W. Larson

1513 M. R. Baer

1520 C. W. Peterson 
1530 L. W. Davison

1531 S. L. Thompson

1531 T. K. Bergstresser

1531 M. Elrick

1531 L. N. Kmetyk

1531 J. M. McGlaun

1531 S. L. Passman

1531 J. S. Rottler

1531 J. W. Swegle

1533 P. Yarrington

1533 W. T. Brown

1533 P. J. Chen

1533 W. R. Davey

1533 A. V. Farnsworth

1533 G. I. Kerley

1533 M. E. Kipp

1533 S. T. Montgomery

1533 F. R. Norwood

1533 J. K. Prentice

1533 A. C. Robinson (20)

1534 J. R. Asay

1534 L. M. Barker

1534 L. C. Chhabildas

1534 J. E. Dunn

1534 D. E. Grady

$1534 \mathrm{M}$. Shahinpoor

1534 T. G. Trucano

1534 J. L. Wise

1550 R. C. Maydew

3141 S. A. Landenberger (5)

3151 W. L. Garner (3)

3154-3 C. H. Dalin (8)

For DOE/TIC

$5100 \mathrm{H}$. W. Schmitt

5160 G. R. Otey

5164 J. F. Cuderman

5164 H. P. Fell

5164 S. W. Hatch

8024 P. M. Dean

8400 R. C. Wayne

8430 J. Vitko

8434 J. B. Woodard

8434 T. E. Owen

8434 C. J. Pignolet
8434 C. A. Pura

8470 R. L. Rinne

8478 M. E. John

9014 J. W. Keizur

9014 R. J. Lawrence

9014 R. M. Zazworsky

9015 R. R. Preston

9122 M. J. Forrestal 